\title{
SUB-GAUSSIAN ESTIMATES OF HEAT KERNELS ON INFINITE GRAPHS
}

\section{ALEXANDER GRIGOR'YAN AND ANDRAS TELCS}

\begin{abstract}
We prove that a two-sided sub-Gaussian estimate of the heat kernel on an infinite weighted graph takes place if and only if the volume growth of the graph is uniformly polynomial and the Green kernel admits a uniform polynomial decay.
\end{abstract}

\section{Contents}

1. Introduction . . . . . . . . . . . . . . . . . . . 4 452

2. Statement of the main result . . . . . . . . . . . . . . . . . . . . . . . . . . . . . . . . . . .

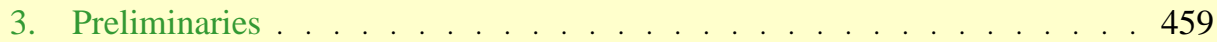

4. Outline of the proof and its consequences . . . . . . . . . . . . . . . 464

5. The Faber-Krahn inequality and on-diagonal upper bounds . . . . . . . . 468

6. The mean exit time and the Green kernel . . . . . . . . . . . . . . . 476

7. Sub-Gaussian term . . . . . . . . . . . . . . . . . . . . . 478

8. Off-diagonal upper bound of the heat kernel . . . . . . . . . . . . . . 482

9. On-diagonal lower bound . . . . . . . . . . . . . . . . . . . . . . . . . . 484

10. The Harnack inequality and the Green kernel . . . . . . . . . . . . . . . . . . . . . . . . . . . . . . . .

11. Oscillation inequalities . . . . . . . . . . . . . . . . . . . 488

12. Time derivative of the heat kernel . . . . . . . . . . . . . . . . . 490 490

13. Off-diagonal lower bound . . . . . . . . . . . . . . . . . . . 493

14. Parity matters . . . . . . . . . . . . . . . . . . . . . . 499

15. Consequences of the heat kernel estimates . . . . . . . . . . . . . . . . . . . . . . . . . . . . . .

Appendix. The list of the lettered conditions . . . . . . . . . . . . . . . . . . . . . . . . . . . . . . . . . .

References . . . . . . . . . . . . . . . . . . 506

DUKE MATHEMATICAL JOURNAL

Vol. 109, No. 3, (C) 2001

Received 5 July 2000. Revisions received 18 October 2000.

2000 Mathematics Subject Classification. Primary 60B99, 60J35, 60G50.

Grigor'yan's work supported by Engineering and Physical Sciences Research Council research fellowship number B/94/AF/1782.

Telcs's work partially supported by a visiting grant of the London Mathematical Society. 


\section{Introduction}

Consider the heat equation

$$
\frac{\partial f}{\partial t}=\Delta f
$$

where $f=f(t, x)$ is a function of $t>0$ and $x \in \mathbb{R}^{n}$, and where $\Delta$ is the Laplace operator in $\mathbb{R}^{n}$. The fundamental solution to (1.1) is given by the classical GaussWeierstrass formula

$$
f(t, x)=\frac{1}{(4 \pi t)^{n / 2}} \exp \left(-\frac{|x|^{2}}{4 t}\right) .
$$

The function $p_{t}(x, y)=f(t, x-y)$ is called the heat kernel of the Laplace operator.

In the past three decades, there have been numerous works devoted to estimates of heat kernels in various settings (see, e.g., books and surveys [4], [15], [16], [25], [35], [52], [55], [65], [66]). These are parabolic equations with variable coefficients, the heat equation on Riemannian manifolds, the discrete heat equation on graphs, and the heat semigroups on general metric measure spaces including fractal-like sets. Despite the high diversity of the underlying spaces and equations, in many important cases the heat kernel is naturally defined and, moreover, admits the so-called Gaussian estimates.

For any metric measure space $M$ with distance $d$ and measure $\mu$, denote by $B(x, r)$ the open metric ball of radius $r$ centered at $x$, and denote by $V(x, r)$ its measure $\mu$. Suppose first that $M$ is either a discrete group or a Lie group, with properly defined $d, \mu$ and the heat kernel $p_{t}(x, y)$. Assume that the volume growth of $M$ is polynomial; that is, for some $\alpha>0$,

$$
V(x, r) \simeq r^{\alpha} .
$$

(Here the sign $\simeq$ means that the ratio of both sides of (1.2) stays between two positive constants.) Then the heat kernel on $M$ admits the following Gaussian estimate (see [64], [37]):

$$
p_{t}(x, y) \simeq t^{-\alpha / 2} \exp \left(-\frac{d^{2}(x, y)}{c t}\right)
$$

(where the positive constant $c$ may be different for the upper and lower bounds). The heat kernel in $\mathbb{R}^{n}$ obviously satisfies (1.3) with $\alpha=n$.

Suppose now that $M$ is a complete manifold with nonnegative Ricci curvature. Then the following estimate of P. Li and S.-T. Yau [47] is well known:

$$
p_{t}(x, y) \simeq \frac{1}{V(x, \sqrt{t})} \exp \left(-\frac{d^{2}(x, y)}{c t}\right) .
$$

In particular, if $V(x, r) \simeq r^{\alpha}$, then the heat kernel again satisfies the estimate (1.3). 
As we see, for groups of polynomial growth and for nonnegatively curved manifolds, the heat kernel is fully determined (up to constant factors) by the volume growth function. In other words, the potential theory on such spaces is characterized by a single parameter $\alpha$-the exponent of the volume growth.

The presence of Gaussian estimates (1.3) or (1.4) reflects certain properties of the space $M$. In particular, (1.4) implies that the Markov process $X_{t}$ with the transition density $p_{t}(x, y)$ has the diffusion speed of the order $t^{1 / 2}$. The latter means that the process $X_{t}$, started at a point $x$, first exits the ball $B(x, R)$ at the time $t \simeq R^{2}$.

The development of Markov processes on fractals and fractal-like graphs (see [10], [7], [30], [36], [40], [41], [42], [44], [45], [59], [67], etc.) has led to construction of homogeneous metric spaces $M$ where the process $X_{t}$ has the diffusion speed of the order $t^{1 / \beta}$, with some $\beta>2$. Such a process $X_{t}$ is referred to as subdiffusive, and it is characterized by two parameters $\alpha$ and $\beta$, which determine sub-Gaussian estimates of the heat kernel:

$$
p_{t}(x, y) \simeq t^{-\alpha / \beta} \exp \left(-\left(\frac{d^{\beta}(x, y)}{c t}\right)^{1 /(\beta-1)}\right) .
$$

Here $\alpha$ is the exponent of the volume growth as in (1.2). The Gaussian estimate (1.3) is a particular case of (1.5) for $\beta=2$.

M. Barlow and R. Bass [7] showed that sub-Gaussian estimates (1.5) with $\beta>$ 2 can take place not only on singular spaces such as fractals but also on smooth Riemannian manifolds, for a certain range of time. Similar estimates hold for random walks on certain fractal-like graphs (see [8], [39]). It has become apparent that a large and interesting class of homogeneous spaces features sub-Gaussian estimates of the heat kernel. The potential theory on such spaces is determined by the two parameters and hence cannot be recovered only from the volume growth.*

A natural question arises:

How do we characterize those spaces that admit sub-Gaussian estimates (1.5) of the heat kernel?

If $M$ is a complete noncompact Riemannian manifold, then the validity of Gaussian estimate (1.3) is known to be equivalent to the following two conditions: volume growth (1.2) and the Poincaré inequality

$$
\lambda_{1}^{(N)}(B(x, r)) \geq \frac{c}{r^{2}},
$$

where $\lambda_{1}^{(N)}(B)$ is the first nonzero eigenvalue of the Neumann boundary value problem in the ball $B$ (see [53], [31]; similar results are known also for graphs (see [28])

*The parameters $\alpha$ and $\beta$ must satisfy the inequalities $2 \leq \beta \leq \alpha+1$, which seem to be the only constraints on $\alpha$ and $\beta$. We are indebted to Martin Barlow for providing us with the evidence for the latter. 
and for abstract local Dirichlet spaces (see [57])). It may be tempting to conjecture that by replacing $r^{2}$ by $r^{\beta}$ in (1.6), one obtains equivalent conditions for sub-Gaussian estimates. However, this conjecture is false. At the present time, no similar characterization of spaces with sub-Gaussian estimates seems to be known. All examples of spaces where (1.5) is proved are fractal-like spaces featuring a self-similarity structure.

The purpose of this paper is to provide a new approach to obtaining sub-Gaussian estimates of the heat kernel. Our point of departure is the understanding that, apart from the uniform volume growth $V(x, r) \simeq r^{\alpha}$, we have to introduce additional hypotheses, which would contain the second parameter $\beta$ and provide the necessary homogeneity of the space. (Just the uniform volume growth is not enough for the latter.)

Let $g(x, y)$ be the Green kernel on $M$; that is,

$$
g(x, y)=\int_{0}^{\infty} p_{t}(x, y) d t .
$$

Recall that, in $\mathbb{R}^{n}, g(x, y)=c_{n}|x-y|^{-(n-2)}$ if $n>2$ and $g \equiv \infty$ if $n \leq 2$.

Our general result says the following:

Given the parameters $\alpha>\beta \geq 2$, the two-sided sub-Gaussian estimate

$$
p_{t}(x, y) \simeq t^{-\alpha / \beta} \exp \left(-\left(\frac{d^{\beta}(x, y)}{c t}\right)^{1 /(\beta-1)}\right)
$$

holds if and only if

$$
V(x, r) \simeq r^{\alpha} \quad \text { and } \quad g(x, y) \simeq d(x, y)^{-(\alpha-\beta)} .
$$

We do not specify here the ranges of the variables $x, y, t, r$ because they are different for different settings. In the present paper, we treat the case when the underlying space is a graph, and the time is also discrete. However, the graph case already contains all difficulties. We present the proof in such a way that only minimal changes are required to pass to a general setting of abstract metric spaces, which will be dealt with elsewhere. The exact statements are given in Section 2. Note that our result is new, even for the Gaussian case $\beta=2$.

Hypothesis (1.8) consists of two conditions of different nature. The first one is a geometric condition of the volume growth, whereas the second is an estimate of a fundamental solution to an elliptic equation. Neither of them separately implies heat kernel bounds (1.7). Surprisingly enough, the exponent $\beta$, which provides the scaling of space and time variables for a parabolic equation, can be recovered from an elliptic equation, although combined with the volume growth.

The paper is arranged as follows. In Section 2 we state the main result-Theorem 2.1. In Section 3 we introduce necessary tools such as the discrete Laplace operator, 
its eigenvalues, the mean exit time, and so on. In Section 4 we describe the scheme of the proof of Theorem 2.1, as well as some consequences. In particular, we mention some other conditions equivalent to (1.7). The actual proof of Theorem 2.1 consists of many steps that are considered in detail in Sections 5-15.

Notation. The letters $c, C$ are reserved for positive constants not depending on the variables in question. They may be different on different occurrences, even within the same formula. All results of the paper are quantitative in the sense that the constants in conclusions depend only on the constants in hypotheses.

The relation $f \simeq g$ means that the ratio of functions $f$ and $g$ is bounded from above and below by positive constants, for the specified range of the variables. If one of those functions contains a sub-Gaussian factor $\exp \left(-\left(d^{\beta} /(c t)\right)^{1 /(\beta-1)}\right)$, then the constant $c$ in exp may be different for the upper and lower bounds (cf. (1.7)).

We use a number of lettered formulas such as $(U E),(L E)$, and so on, to refer to the most important and frequently used conditions. In the appendix we provide a complete list of all such formulas.

\section{Statement of the main result}

Throughout the paper, $\Gamma$ denotes an infinite, connected, locally finite graph. If $x, y \in$ $\Gamma$, then we write $x \sim y$, provided $x$ and $y$ are connected by an edge. The graph is always assumed nonoriented; that is, $x \sim y$ is equivalent to $y \sim x$. We do not exclude loops so that $x \sim x$ is possible. If $x \sim y$, then $\overline{x y}$ denotes the edge connecting $x$ and $y$. The distance $d(x, y)$ is the minimal number of edges in any edge path connecting $x$ and $y$.

Assume that graph $\Gamma$ is endowed by a weight $\mu_{x y}$, which is a symmetric nonnegative function on $\Gamma \times \Gamma$ such that $\mu_{x y}>0$ if and only if $x \sim y$. Given $\mu_{x y}$, we also define a measure $\mu$ on vertices by

$$
\mu(x):=\sum_{y \sim x} \mu_{x y}
$$

and

$$
\mu(A):=\sum_{x \in A} \mu(x),
$$

for any finite set $A \subset \Gamma$. The couple $(\Gamma, \mu)$ is called a weighted graph. Here $\mu$ refers both to the weight $\mu_{x y}$ and to the measure $\mu$.

Any graph $\Gamma$ admits a standard weight, which is defined by $\mu_{x y}=1$ for all edges $\overline{x y}$. For such a weight, $\mu(x)$ is equal to the degree of the vertex $x$, which is the number of its neighbors. 
Any weighted graph has a natural Markov operator $P(x, y)$ defined by

$$
P(x, y):=\frac{\mu_{x y}}{\mu(x)} .
$$

Clearly, we have

$$
\sum_{y \in \Gamma} P(x, y)=1
$$

and

$$
P(x, y) \mu(x)=P(y, x) \mu(y) .
$$

For the Markov operator $P$, there is an associated random walk $X_{n}$, jumping at each time $n \in \mathbb{N}$ from a current vertex $x$ to a neighboring vertex $y$ with probability $P(x, y)$. The process $X_{n}$ is Markov and reversible with respect to measure $\mu$. If $\mu$ is the standard weight on $\Gamma$, then $X_{n}$ is called a simple random walk on $\Gamma$.

Conversely, given a countable set $\Gamma$ with a measure $\mu$ and a Markov operator $P(x, y)$ on $\Gamma$ satisfying (2.3), identity (2.1) uniquely determines a symmetric weight $\mu_{x y}$ on $\Gamma \times \Gamma$. Then one defines edges $\overline{x y}$ as those pairs of vertices for which $\mu_{x y} \neq 0$, and one obtains a weighted graph $(\Gamma, \mu)$. One has to assume in addition that the resulting graph $\Gamma$ is connected and locally finite.

Let $P_{n}$ denote the $n$th convolution power of the operator $P$. Alternatively, $P_{n}(x, y)$ is the transition function of the random walk $X_{n}$; that is,

$$
P_{n}(x, y)=\mathbb{P}_{x}\left(X_{n}=y\right) .
$$

Define also the transition density of $X_{n}$, or the heat kernel, by

$$
p_{n}(x, y):=\frac{P_{n}(x, y)}{\mu(y)} .
$$

As obviously follows from (2.3), $p_{n}(x, y)=p_{n}(y, x)$.

The only a priori assumption that we normally make about the transition probability is the following:

$$
P(x, y) \geq p_{0}, \quad \forall x \sim y,
$$

where $p_{0}$ is a positive constant. Due to (2.2), hypothesis $\left(p_{0}\right)$ implies that the degree of each vertex $x \in \Gamma$ is uniformly bounded from above. The latter is in fact equivalent to $\left(p_{0}\right)$, provided $X_{n}$ is a simple random walk.

By sub-Gaussian heat kernel estimates on graphs, we mean the following inequalities:

$$
p_{n}(x, y) \leq C n^{-\alpha / \beta} \exp \left(-\left(\frac{d(x, y)^{\beta}}{C n}\right)^{1 /(\beta-1)}\right)
$$


and

$$
p_{n}(x, y)+p_{n+1}(x, y) \geq c n^{-\alpha / \beta} \exp \left(-\left(\frac{d(x, y)^{\beta}}{c n}\right)^{1 /(\beta-1)}\right), \quad n \geq d(x, y),
$$

where $x, y$ are arbitrary points on $\Gamma$ and where $n$ is a positive integer.

Let us comment on the differences between $(U E)$ and $(L E)$. First, observe that $p_{n}(x, y)=0$ whenever $n<d(x, y)$. (Indeed, the random walk cannot get from $x$ to $y$ in a number of steps smaller than $d(x, y)$.) Therefore, the restriction $n \geq d(x, y)$ in $(L E)$ is necessary. We could assume the same restriction in $(U E)$, but if $p_{n}(x, y)=$ 0 , then $(U E)$ is true anyway. Another difference-using $p_{n}+p_{n+1}$ in $(L E)$ in place of $p_{n}$ in $(U E)$ - is due to the parity problem. Indeed, if graph $\Gamma$ is bipartite (e.g., $\left.\mathbb{Z}^{D}\right)$, then $p_{n}(x, y)=0$ whenever $n$ and $d(x, y)$ have different parities. Therefore, the lower bound for $p_{n}$ cannot hold in general, and we state it for $p_{n}+p_{n+1}$ instead. Alternatively, one could say that the lower bound holds either for $p_{n}$ or for $p_{n+1}$. The structure of the graph may cause one of $p_{n}, p_{n+1}$ to be small (or even vanish), but it is not possible to decide a priori which of these two terms admits the lower bound (see Section 14 for more details).

Denote by $B(x, R)$ a ball on $\Gamma$ of radius $R$ centered at $x$, and denote by $V(x, R)$ its measure, that is,

$$
B(x, R):=\{y \in \Gamma: d(x, y)<R\}, \quad V(x, R):=\mu(B(x, R)) .
$$

We say that graph $(\Gamma, \mu)$ has the regular volume growth of degree $\alpha$ if

$$
V(x, R) \simeq R^{\alpha}, \quad \forall x \in \Gamma, R \geq 1 .
$$

The Green kernel of $(\Gamma, \mu)$ is defined by

$$
g(x, y):=\sum_{n=0}^{\infty} p_{n}(x, y) .
$$

Assuming that $\alpha>\beta$, the estimates $(U E)$ and $(L E)$ imply, upon summation in $n$,

$$
g(x, y) \simeq d(x, y)^{-\gamma}, \quad \forall x \neq y,
$$

where $\gamma=\alpha-\beta$. It turns out that $(G)$, together with the volume growth condition $(V)$, is sufficient to recover the heat kernel estimates $(U E)$ and $(L E)$, as is stated in the following main theorem.

\section{THEOREM 2.1}

Let $\alpha>\beta>1$, and let $\gamma=\alpha-\beta$. For any infinite connected weighted graph $(\Gamma, \mu)$ satisfying $\left(p_{0}\right)$, the following equivalence holds:

$$
(V)+(G) \Longleftrightarrow(U E)+(L E) .
$$




\section{Remark 2.1}

Under hypotheses $(V)$ and $(G)$, some partial heat kernel estimates were obtained by A. Telcs [62].

It is well known that a simple random walk in $\mathbb{Z}^{D}$ admits the Gaussian estimate

$$
c n^{-D / 2} \exp \left(-\frac{d^{2}(x, y)}{c n}\right) \leq p_{n}(x, y) \leq C n^{-D / 2} \exp \left(-\frac{d^{2}(x, y)}{C n}\right),
$$

subject to the restrictions $n \equiv d(x, y)(\bmod 2)$ and $d(x, y) \leq n$. Similar Gaussian estimates were also proved for more general graphs, under various assumptions (see [37], [54], [22], [28]). It is easy to see that (2.4) is equivalent to $(U E)+(L E)$ for $\alpha=D$ and $\beta=2$ (see Section 14 for the parity matters).

Barlow and Bass [8] constructed a family of graphs—graphical Sierpiński carpets (resembling in the large scale the multi-dimensional Sierpiński carpet), which are characterized by the parameters $\alpha$ and $\beta$. Heat kernels on those graphs satisfy subGaussian estimates $(U E)$ and $(L E)$. In general, the parameters $\alpha$ and $\beta$ in $(U E)$ and $(L E)$ must satisfy the inequalities

$$
2 \leq \beta \leq \alpha+1,
$$

which can be seen as follows. By [9, Th. 2.1], the lower bound in $(V)$ implies the ondiagonal upper bound $p_{n}(x, x) \leq C n^{-\alpha /(\alpha+1)}$. By the result of [48], the upper bound in $(V)$ implies the on-diagonal lower bound $p_{n}(x, x) \geq c(n \log n)^{-\alpha / 2}$. Comparing these estimates with the on-diagonal lower and upper bounds implied by $(L E)$ and $(U E)$, we obtain (2.5) (cf. [4, Th. 3.20 and Rem. 3.22], [59], as well as Lemma 5.4).

The sub-Gaussian estimates for different $\alpha$ and $\beta$ are related as follows. Consider the right-hand side of $(U E)$ and $(L E)$ as a function of $\alpha$ and $\beta$. It is easy to see that it decreases as $\beta$ and $\alpha / \beta$ simultaneously increase (assuming $d(x, y) \geq n$ ). In particular, $(U E)$ gets stronger (and $(L E)$ gets weaker) on increasing of $\alpha$ with constant $\beta$, whereas in general there is no monotonicity in $\beta$.

Estimates $(U E)$ and $(L E)$ were proved by O. Jones [39] for the graphical Sierpiński gasket. The latter is a graph that is obtained from an equilateral triangle by a fractal-like construction (see Figure 1). The reason for a subdiffusive behaviour of the random walk on such graphs is that they contain plenty of "holes" of all sizes, which causes the random walk to spend more time on circumventing the obstacles rather than on moving away from the origin.

It is possible to show that $(V)$ and $(G)$ imply $\beta \geq 2$ (see Lemma 5.4). The assumption $\alpha>\beta$ is necessary to ensure the finiteness of the Green function. It is known that either $g(x, y)$ is finite for all $x, y$ or $g \equiv \infty$. In the first case the graph 


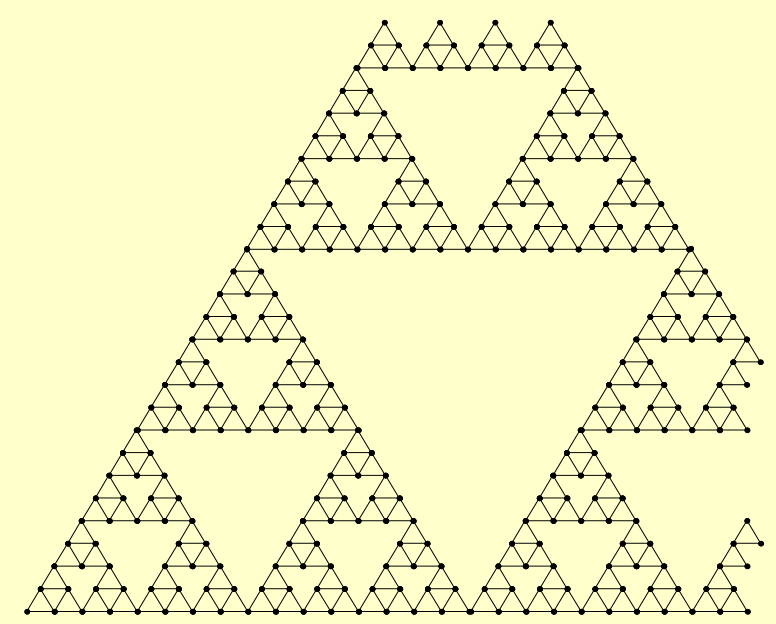

Figure 1. A fragment of the graphical Sierpiński gasket

$(\Gamma, \mu)$ is called transient and in the second case recurrent (e.g., $\mathbb{Z}^{D}$ is transient if $D \geq 3$ and recurrent otherwise). Hence, Theorem 2.1 serves only transient graphs.

The question of finding equivalent conditions for sub-Gaussian estimates $(U E)$ and $(L E)$ is equally interesting for recurrent graphs. Note that the graph in Figure 1 is recurrent.* Indeed, the volume function on this graph obviously admits the estimate

$$
V(x, r) \leq C r^{2},
$$

which implies the recurrence (see [18], [66]). Alternatively, one can see directly that $\alpha<\beta$ because the parameters $\alpha$ and $\beta$ for the Sierpiński gasket are $\alpha=\log 3 / \log 2$ and $\beta=\log 5 / \log 2$ (see [4]).

Some hints on the recurrent case are given in Section 4.

\section{Preliminaries}

If $P$ is the Markov operator of a weighted graph $(\Gamma, \mu)$ and if $I$ is the identity operator, then $\Delta:=P-I$ is called the Laplace operator of $(\Gamma, \mu)$. For any set $A \subset \Gamma$, denote by $\bar{A}$ the set containing all vertices of $A$ and all their neighbors. If a function $f$ is defined on $\bar{A}$, then $\Delta f$ is defined on $A$ and

$$
\Delta f(x)=\sum_{y \sim x} P(x, y) f(y)-f(x)=\frac{1}{\mu(x)} \sum_{y \in \Gamma}\left(\nabla_{x y} f\right) \mu_{x y},
$$

*Plenty of examples of transient graphs and fractals with sub-Gaussian heat kernel bounds can be found in [4], [7], and [8]. 
where

$$
\nabla_{x y} f:=f(y)-f(x) .
$$

Note that although the summation in the second sum in (3.1) runs over all vertices $y$, the summand is nonvanishing only if $y \sim x$.

The following is a discrete analogue of the Green formula: for any finite set $A$ and for all functions $f$ and $g$ defined on $\bar{A}$,

$$
\sum_{x \in A} \Delta f(x) g(x) \mu(x)=\sum_{x \in A, y \notin A}\left(\nabla_{x y} f\right) g(x) \mu_{x y}-\frac{1}{2} \sum_{x, y \in A}\left(\nabla_{x y} f\right)\left(\nabla_{x y} g\right) \mu_{x y} .
$$

We say that a function $v$ is harmonic in set $A$ if $v$ is defined in $\bar{A}$ and $\Delta v=0$ in $A$. Similarly, we say that a function $v$ is superharmonic if $\Delta v \leq 0$. Observe that the inequality $\Delta v \leq 0$ is equivalent to

$$
v(x) \geq \sum_{y \sim x} P(x, y) v(y) .
$$

The latter implies, in particular, that the infimum of a family of superharmonic functions is again superharmonic.

For any nonempty set $A \subset \Gamma$, let $c_{0}(A)$ be the set of functions on $\Gamma$ whose support is finite and is in $A$. Denote by $\Delta_{A}$ the Laplace operator with the vanishing Dirichlet boundary condition on $A$; that is,

$$
\Delta_{A} f(x):= \begin{cases}\Delta f, & x \in A, \\ 0, & x \notin A .\end{cases}
$$

The operator $\Delta_{A}$ is symmetric with respect to the measure $\mu$ and is nonpositive definite. Moreover, it is essentially self-adjoint in $L^{2}(A, \mu)$.

For a finite set $A$, denote by $|A|$ its cardinality. If $A$ is finite and nonempty, then the operator $-\Delta_{A}$ has $|A|$ nonnegative eigenvalues that we enumerate in increasing order and denote as follows:

$$
\lambda_{1}(A) \leq \lambda_{2}(A) \leq \cdots \leq \lambda_{|A|}(A) .
$$

It is known that all eigenvalues $\lambda_{i}(A)$ lie in the interval $[0,2]$ and that $\lambda_{1}(A) \in[0,1]$ (see, e.g., [19], [22, Sec. 3.3]). The smallest eigenvalue $\lambda_{1}(A)$ admits the variational definition

$$
\lambda_{1}(A)=\inf _{f \in c_{0}(A)} \frac{-(\Delta f, f)}{(f, f)}=\inf _{f \in c_{0}(A)} \frac{(1 / 2) \sum_{x \sim y}\left(\nabla_{x y} f\right)^{2} \mu_{x y}}{\sum_{x} f^{2}(x) \mu(x)},
$$

where

$$
(f, g):=\sum_{x \in \Gamma} f(x) g(x) \mu(x)
$$


If $A=B(x, R)$, then we write, for simplicity,

$$
\lambda(x, R):=\lambda_{1}(B(x, R)) .
$$

Given a nonempty set $A \subset \Gamma$, let $X_{n}^{A}$ be the random walk on $(\Gamma, \mu)$ with the killing condition outside $A$. Its Markov operator $P^{A}(x, y)$ is defined by

$$
P^{A}(x, y):= \begin{cases}P(x, y), & x, y \in A, \\ 0, & \text { otherwise. }\end{cases}
$$

The transition function $P_{n}^{A}(x, y)$ of $X_{n}^{A}$ is defined inductively: $P_{0}^{A}(x, y)=\delta_{x y}$ and

$$
P_{n+1}^{A}(x, y)=\sum_{z \in \Gamma} P_{n}^{A}(x, z) P^{A}(z, y)=\sum_{z \in \Gamma} P^{A}(x, z) P_{n}^{A}(z, y) .
$$

As easily follows from (3.4), the function $u_{n}(x)=P_{n}^{A}(x, y)$ satisfies in $A \times \mathbb{N}$ the discrete heat equation

$$
u_{n+1}-u_{n}=\Delta_{A} u_{n} .
$$

The heat kernel $p_{n}^{A}(x, y)$ of $X_{n}^{A}$ is defined by

$$
p_{n}^{A}(x, y):=\frac{P_{n}^{A}(x, y)}{\mu(y)} .
$$

As follows from (2.1), $p^{A}$ is symmetric in $x$ and $y$. In particular, the kernel $p_{n}^{A}(x, y)$ satisfies heat equation (3.5) both in $(n, x)$ and $(n, y)$. If $f(x)$ is a function on $A$, then the function

$$
u_{n}(x):=P_{n}^{A} f(x)=\sum_{y \in A} p_{n}^{A}(x, y) f(y) \mu(y)
$$

solves, in $A \times \mathbb{N}$, heat equation (3.5) with initial data $u_{0}=f$ and boundary data $u_{n}(x)=0$ if $x \notin A$.

The Green function of $X_{n}^{A}$ is defined by

$$
G_{A}(x, y):=\sum_{n=0}^{\infty} P_{n}^{A}(x, y) .
$$

The alternative definition is that the function $G_{A}(x, y)$ is the infimum of all positive fundamental solutions of the Laplace equation in $A$. If the Green function is finite, then, for any $y \in A$, we have $\Delta_{A} G_{A}(\cdot, y)=-\delta_{y}$. The opposite case, when $G_{A}(x, y) \equiv+\infty$, is equivalent to the recurrence of the process $X_{n}^{A}$.

The Green kernel $g_{A}(x, y)$ is defined by

$$
g_{A}(x, y)=\frac{G_{A}(x, y)}{\mu(y)}=\sum_{n=0}^{\infty} p_{n}^{A}(x, y) .
$$


Clearly, the Green kernel is symmetric in $x, y$. Therefore, if $g_{A}$ is finite, then $g_{A}$ is superharmonic in $A$ with respect to both $x$ and $y$, and it is harmonic away from the diagonal $x=y$. Observe that if $\mu(x) \simeq 1$ (which in particular follows from $(V)$ ), then $G_{A}(x, y) \simeq g_{A}(x, y)$ and $p_{n}^{A}(x, y) \simeq P_{n}^{A}(x, y)$.

It is easy to see that kernels $p_{n}^{A}(x, y)$ and $g_{A}(x, y)$ increase when $A$ is enlarged and tend to global kernels $p_{n}(x, y)$ and $g(x, y)$ (defined in Section 2) as an increasing sequence of sets $A$ exhausts $\Gamma$.

If $A$ is finite and nonempty, then it makes sense to consider the Dirichlet problem in $A$,

$$
\begin{cases}\Delta u=f & \text { in } A, \\ u=h & \text { in } \bar{A} \backslash A,\end{cases}
$$

where $f$ and $h$ are given function on $A$ and $\bar{A} \backslash A$, respectively. As follows easily from the maximum principle, the solution $u$ exists and is unique. For a finite set $A, c_{0}(A)$ is identified with all functions on $A$ extended by zero outside $A$. Then the equation

$$
\Delta_{A} u=f,
$$

where $u$ and $f$ are in $c_{0}(A)$, is equivalent to Dirichlet problem (3.6) with $h=0$. Its solution is given by means of the Green operator $G_{A}$ as follows:

$$
u(x)=-G_{A} f(x)=-\sum_{y} G_{A}(x, y) f(y) .
$$

In other words, we have $G_{A}=\left(-\Delta_{A}\right)^{-1}$.

For any set $A \subset \Gamma$ and a point $x \in \Gamma$, define the mean exit time $E_{A}(x)$ by

$$
E_{A}(x):=\sum_{y \in A} G_{A}(x, y) .
$$

As follows from the above discussion, the function $E_{A}(x)$ solves the following boundary value problem in $A$ :

$$
\begin{cases}\Delta u=-1 & \text { in } A, \\ u=0 & \text { outside } A .\end{cases}
$$

Denote by $T_{A}$ the first exit time from set $A$ for the process $X_{n}$; that is,

$$
T_{A}:=\min \left\{k: X_{k} \notin A\right\} .
$$

We claim that $E_{A}(x)=\mathbb{E}_{x}\left(T_{A}\right)$, which justifies the term "mean exit time" for $E_{A}$. Indeed, $T_{A}$ coincides with the cardinality of all $n=0,1,2, \ldots$ for which $X_{n}^{A}$ is in $A$; that is,

$$
T_{A}=\sum_{n=0}^{\infty} \mathbf{1}_{\left\{X_{n}^{A} \in A\right\}},
$$


whence

$$
\mathbb{E}_{x}\left(T_{A}\right)=\sum_{n=0}^{\infty} \mathbb{P}_{x}\left(X_{n}^{A} \in A\right)=\sum_{n=0}^{\infty} \sum_{y \in A} P_{n}^{A}(x, y)=\sum_{y \in A} G_{A}(x, y)=E_{A}(x) .
$$

If $A=B(x, R)$, then we use a shorter notation

$$
E(x, R):=E_{B(x, R)}(x) .
$$

Another function associated with the exit time is the exit probability, defined by

$$
\Psi_{n}^{A}(x):=\mathbb{P}_{x}\left\{X_{k} \notin A \text { for some } k \leq n\right\}=\mathbb{P}_{x}\left\{T_{A} \leq n\right\} .
$$

In other words, $\Psi_{n}^{A}(x)$ is the probability that the random walk $X_{k}$ started at $x$ will at least once exit $A$ by time $n$. Alternatively, $\Psi_{n}^{A}(x)$ can be defined as the solution $u_{n}(x)$ to the following initial boundary value problem in $A \times \mathbb{N}$ :

$$
\begin{cases}u_{n+1}-u_{n}=\Delta u_{n}, & \\ u_{0}(x)=0, & x \in A, \\ u_{n}(x)=1, & x \notin A \text { and } n \geq 0 .\end{cases}
$$

If $A=B(x, R)$, then we use the shorter notation

$$
\Psi_{n}(x, R):=\Psi_{n}^{B(x, R)}(x) .
$$

To conclude this section, we prove two useful consequences of condition $\left(p_{0}\right)$ :

$$
P(x, y) \geq p_{0}, \quad \forall x \sim y .
$$

PROPOSITION 3.1

If $\left(p_{0}\right)$ holds, then, for all $x \in \Gamma$ and $R>0$ and for some $C=C\left(p_{0}\right)$,

$$
V(x, R) \leq C^{R} \mu(x) \text {. }
$$

Remark 3.1

Inequality (3.12) implies that, for a bounded range of $R, V(x, R) \simeq \mu(x)$.

\section{Proof}

Let $x \sim y$. Since $P(x, y)=\mu_{x y} / \mu(x)$ and $\mu_{x y} \leq \mu(y)$, hypothesis $\left(p_{0}\right)$ implies $p_{0} \mu(x) \leq \mu(y)$. Similarly, $p_{0} \mu(y) \leq \mu(x)$. Iterating these inequalities, we obtain, for arbitrary $x$ and $y$,

$$
p_{0}^{d(x, y)} \mu(y) \leq \mu(x) .
$$

Another consequence of $\left(p_{0}\right)$ is that any point $x$ has at most $p_{0}^{-1}$ neighbors. Therefore, any ball $B(x, R)$ has at most $C^{R}$ vertices inside. By (3.13), any point $y \in B(x, R)$ has measure at most $p_{0}^{-R} \mu(x)$, whence (3.12) follows. 
PROPOSITION 3.2

Assume that hypothesis $\left(p_{0}\right)$ holds on $(\Gamma, \mu)$. Let function $v$ be nonnegative in $\bar{A}$ and superharmonic in A. Then, for all points $x, y \in A$ such that $x \sim y$, we have $v(x) \simeq v(y)$.

Proof

Indeed, the superharmonicity of $v$ implies

$$
v(x) \geq \sum_{z \sim x} P(x, z) v(z) \geq P(x, y) v(y),
$$

whence $v(x) \geq p_{0} v(y)$ by $\left(p_{0}\right)$. In the same way, $v(y) \geq p_{0} v(x)$, whence the claim follows.

\section{Outline of the proof and its consequences}

The proof of Theorem 2.1 consists of many steps. Here we describe the logical order of these steps. The rest of the paper is arranged so that each section treats a certain topic corresponding to one or more steps in the proof of Theorem 2.1.

Apart from conditions $(V),(G),(U E)$, and $(U E)$ described in Section 2, we introduce here some more lettered conditions that are widely used in the proof.

We say that the Faber-Krahn inequality holds on $(\Gamma, \mu)$ if, for some positive exponent $v$,

$$
\lambda_{1}(A) \geq c \mu(A)^{-1 / v}
$$

for all nonempty finite sets $A \subset \Gamma$. In particular, $(F K)$ holds in $\mathbb{Z}^{D}$ with $v=D / 2$. If $\Gamma$ is infinite and connected and if $\mu$ is the standard weight on $\Gamma$, then $(F K)$ automatically holds with $v=1 / 2$ (see [9, Prop. 2.5]). We are interested in $(F K)$ with $v=\alpha / \beta$, where $\alpha$ and $\beta$ are the parameters from $(U E)$ and $(L E)$, in which case we have $v>1$.

An easy consequence of $(U E)$ is the diagonal upper estimate

$$
p_{n}(x, x) \leq C n^{-\alpha / \beta}
$$

for all $x \in \Gamma$ and $n \geq 1$.

Consider the following estimates for the mean exit time and the exit probability:

$$
E(x, R) \simeq R^{\beta}
$$

for all $x \in \Gamma, R \geq 1$, and

$$
\Psi_{n}(x, R) \leq C \exp \left(-\left(\frac{R^{\beta}}{C n}\right)^{1 /(\beta-1)}\right)
$$

for all $x \in \Gamma, R>0$, and $n \geq 1$. For example, $(E)$ and $(\Psi)$ hold in $\mathbb{Z}^{D}$ with $\beta=2$. 
Part $(V)+(G) \Longrightarrow(U E)$ of Theorem 2.1 is proved by the following chain of implications:

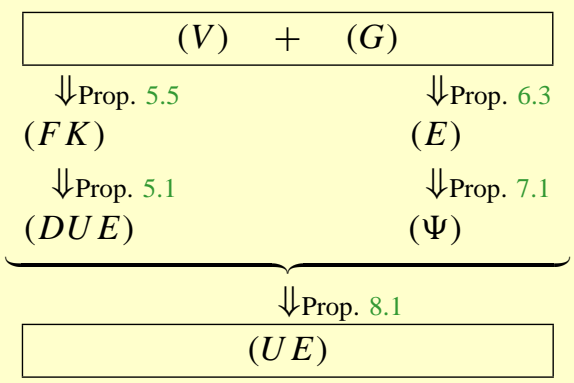

The relations among exponents $\alpha, \beta, \gamma$, and $v$ involved in all conditions are as follows:

$$
\alpha-\beta=\gamma \quad \text { and } \quad \alpha / \beta=v .
$$

Given ( $D U E)$ and $(\Psi)$, one easily obtains full upper bound $(U E)$ using the approach of Barlow and Bass [6] (see Section 8). The method of obtaining Faber-Krahn inequality $(F K)$ from $(V)$ and $(G)$ is based on ideas of G. Carron [14]. The implication $(F K) \Longrightarrow(D U E)$ is a discrete modification of the approach of A. Grigor'yan [32]. The implication $(V)+(G) \Longrightarrow(E)$ was originally proved by Telcs [59], and here we give a simpler proof for that.

The crucial part of the proof of upper estimate $(U E)$ is the implication $(E) \Longrightarrow$ $(\Psi)$. The following nearly Gaussian estimate is true always, without assuming $(E)$ or anything else:

$$
\Psi_{n}(x, R) \leq C \frac{V(x, R)}{\mu(x)} \exp \left(-\frac{R^{2}}{C n}\right)
$$

(see [58] and [33, p. 355]). However, (4.1) is not good enough for us even if we neglect the factor $V(x, R)$ in front of the exponential. Indeed, the range of $n$, for which we apply ( $\Psi$ ), is $n>R$ (see the proof of Proposition 8.1). Assuming $\beta>2$, we have in this range

$$
\left(\frac{R^{\beta}}{n}\right)^{1 /(\beta-1)}>\frac{R^{2}}{n},
$$

so that $(\Psi)$ is stronger than (4.1).

We provide here an entirely new argument for $(E) \Longrightarrow(\Psi)$, which is based on an investigation of solutions of the equation $\Delta v=\lambda v$. Function $v$ can be estimated by comparing it to $\Delta u=-1$ (and the latter is related to the mean exit time). On the other hand, function $(1+\lambda)^{n} v(x)$ satisfies the discrete heat equation and hence can be compared to $\Psi_{n}^{A}(x)$ by using the parabolic comparison principle (see Section 7 for details). Another proof of $(E) \Longrightarrow(\Psi)$ can be obtained by using the probabilistic method of Barlow and Bass [5], [6], [7]. 
Before we consider the proof of lower bound $(L E)$, let us introduce the following conditions.

The near-diagonal lower estimate is

$$
p_{n}(x, y)+p_{n+1}(x, y) \geq c n^{-\alpha / \beta} \quad \text { if } d(x, y) \leq \delta n^{1 / \beta}
$$

for some positive constant $\delta$. Obviously, $(N L E)$ is equivalent to $(L E)$ in the range $d(x, y) \leq \delta n^{1 / \beta}$.

As an intermediate step, we use the following diagonal lower estimate for the killed random walk:

$$
p_{2 n}^{B(x, R)}(x, x) \geq c n^{-\alpha / \beta} \quad \text { if } n \leq \varepsilon R^{\beta}
$$

for some positive constant $\varepsilon$.

We say that the Harnack inequality holds on $(\Gamma, \mu)$ if, for any ball $B(x, 2 R) \subset \Gamma$ and for any nonnegative function $u$ in $\overline{B(x, 2 R)}$ which is harmonic in $B(x, 2 R)$,

$$
\max _{B(x, R)} u \leq H \min _{B(x, R)} u
$$

for some constant $H \geq 1$. The Harnack inequality reflects a certain homogeneity of the graph. For example, it holds for $\mathbb{Z}^{D}$ with the standard weight but fails on the connected sum of two copies of $\mathbb{Z}^{D}$ as well as on a binary tree.

The scheme of the proof of $(V)+(G) \Longrightarrow(L E)$ is shown on the diagram below.

\begin{tabular}{|c|c|c|}
\hline & $(V)+(G)$ & \\
\hline \multicolumn{3}{|c|}{$\Downarrow_{\text {Prop. } 5.5,6.3}$} \\
\hline$(F K)$ & $(E)$ & $(G)$ \\
\hline$\Downarrow_{\text {Prop. } 5.1}$ & $\Downarrow_{\text {Prop. } 7.1}$ & $\Downarrow_{\text {Prop. } 10.1}$ \\
\hline$(D U E)$ & $\underbrace{(\Psi)+(V)}$ & $(H)$ \\
\hline $\begin{array}{c}\Downarrow_{\text {Prop. } 12.3} \\
\text { [deriv] }\end{array}$ & $\begin{array}{c}\Downarrow_{\text {Prop. } 9.1} \\
(D L E)+(E)\end{array}$ & $\begin{array}{l}\Downarrow_{\text {Prop. } 11.2} \\
\text { [osc] }\end{array}$ \\
\hline \multicolumn{3}{|c|}{$\begin{array}{c}\Downarrow_{\text {Prop. } 13.1} \\
(N L E)+(V)\end{array}$} \\
\hline \multicolumn{3}{|c|}{$\Downarrow_{\text {Prop. } 13.2}$} \\
\hline
\end{tabular}
From the previous diagram, we already know that conditions $(F K)$ and $(E)$ follow from $(V)+(G)$, as well as the implications $(F K) \Longrightarrow(D U E)$ and $(E) \Longrightarrow(\Psi)$ :

The central point in the diagram is Proposition 13.1, where $(N L E)$ is obtained from $(D U E),(D L E),(E)$, and $(H)$. The proof goes through the intermediate steps that 
are denoted here by [osc] and [deriv]. The former refers to oscillation inequality (11.3) obtained from $(H)$ in Propositions 11.1 and 11.2, and the latter refers to upper estimate (12.5) for $\left|p_{n+2}-p_{n}\right|$ obtained from ( $D U E$ ) in Proposition 12.3.

The idea of obtaining $(N L E)$ by means of an elliptic Harnack inequality seems to have appeared independently in papers by P. Auscher [2], [3], Barlow and Bass [6], [7], [8], and W. Hebisch and L. Saloff-Coste [38]. Basically, one views the heat equation for the heat kernel as an elliptic equation

$$
\Delta\left(p_{n}+p_{n+1}\right)=f, \quad \text { where } f=p_{n+2}-p_{n} .
$$

The elliptic Harnack inequality and the upper bound for $E(x, r)$ allow one to estimate the oscillation of $p_{n}+p_{n+1}$ via $f$. (In the continuous setting, the latter argument is classical and is due to J. Moser [49].)

On the other hand, the on-diagonal upper bound for $p_{n}$ implies a suitable estimate for the discrete time derivative $p_{n+2}-p_{n}$. The fact that $(D U E)$ implies a certain estimate of the time derivative of the heat kernel is well known. In the context of manifolds it goes back to S. Cheng, Li, and Yau [17] and E. Davies [26], [27] (see also [34]); in the discrete setting it follows from the results of E. Carlen, S. Kusuoka, and D. Stroock [13] and T. Coulhon and Saloff-Coste [23]; and in the setting of fractals it is proved by Barlow and Bass [7].

Having an upper bound for the oscillation of $p_{n}+p_{n+1}$ and the on-diagonal lower bound for $p_{n}+p_{n+1}$, one obtains $(N L E)$. The final step in the proof-the implication $(N L E)+(V) \Longrightarrow(L E)$-is done by using the classical chaining argument of Moser [50] and D. Aronson [1].

The method of obtaining $(D L E)$ from $(\Psi)$ and $(V)$ used in Proposition 9.1 is well known. Its various modifications can be found in [6], [11], [21], [24], [48], [56], and possibly in other places.

The claim that Green kernel estimate $(G)$ implies elliptic Harnack inequality $(H)$ would not surprise experts. In the context of the uniformly elliptic operators in $\mathbb{R}^{D}$, this was first observed by E. Landis [46, p. 145-146] and then was elaborated by N. Krylov and M. Safonov [43] and E. Fabes and Stroock [29]. However, this claim becomes rather nontrivial for arbitrary graphs (and manifolds) because of topological difficulties. We provide here a new, simple, and general proof of the implication $(G) \Longrightarrow(H)$, which is based on the potential theoretic approach of A. Boukricha [12].

Finally, the converse implication $(U E)+(L E) \Longrightarrow(V)+(G)$ is quite straightforward and is proved in Proposition 15.1.

As a consequence of the above diagrams, we see that the following equivalence takes place:

$$
(F K)+(V)+(E)+(H) \Longleftrightarrow(U E)+(L E) .
$$


It is possible to show that this equivalence is also true for recurrent graphs. Furthermore, Faber-Krahn inequality $(F K)$ turns out to follow from $(V)+(E)+(H)$, so that

$$
(V)+(E)+(H) \Longleftrightarrow(U E)+(L E) .
$$

Condition $(H)$ ensures here a necessary homogeneity of the graph, whereas $(V)$ and (E) provide the exponents $\alpha$ and $\beta$, respectively.

Another consequence of the proof is that

$$
(V)+(U E)+(H) \Longleftrightarrow(U E)+(L E)
$$

(see Remark 15.1). There are a number of conditions given in terms of capacities, eigenvalues, and so on, which can replace $(E)$ or $(U E)$ in (4.2) and (4.3), respectively. In the presence of $(V)$ and $(H)$, the purpose of the other condition is to recover the exponent $\beta$ in $(U E)$ and $(L E)$. Note that if $\beta=2$, then $(U E)$ in (4.3) can be replaced by $(D U E)$ (cf. [38]).

The complete proofs of (4.2), (4.3), and other related statements will be given elsewhere.

\section{The Faber-Krahn inequality and on-diagonal upper bounds}

Recall that a Faber-Krahn inequality holds on $(\Gamma, \mu)$ if there are constants $c>0$ and $v>0$ such that, for all nonempty finite sets $A \subset \Gamma$,

$$
\lambda_{1}(A) \geq c \mu(A)^{-1 / \nu} .
$$

We discuss here relationships between eigenvalue estimates like $(F K)$ and estimates of the Green kernel, heat kernel, and volume growth. The outcome is the following implications:

$$
(V)+(G) \Longrightarrow(F K) \Longrightarrow(D U E),
$$

which are contained in Propositions 5.5 and 5.1, respectively, and which constitute a part of the proof of Theorem 2.1.

\section{PROPOSITION 5.1}

Let $(\Gamma, \mu)$ satisfy $\left(p_{0}\right)$, and let $v$ be a positive number. Then the following conditions are equivalent:

(a) Faber-Krahn inequality $(F K)$;

(b) the on-diagonal heat kernel upper bound, for all $x \in \Gamma$ and $n \geq 1$,

$$
p_{n}(x, x) \leq C n^{-v}
$$


(c) the estimate of the level sets of the Green kernel, for all $x \in \Gamma$ and $t>0$,

$$
\mu\{y: g(x, y)>t\} \leq C t^{-\nu /(v-1)},
$$

provided $v>1$.

The analogue of Proposition 5.1 for manifolds was proved by Carron [14]. The equivalence $(a) \Longleftrightarrow(b)$ was also proved in [32] for heat kernels on manifolds, and in [20, Prop. V.1] for random walks satisfying in addition the condition $\inf _{x} P(x, x)>0$.

We provide detailed proof only for the implications $(a) \Longrightarrow(b)$ and $(c) \Longrightarrow(a)$ which we use in this paper. The implication $(b) \Longrightarrow(c)$ can be proved in the following way. By a theorem of N. Varopoulos [63], (DUE) implies a Sobolev inequality. Then one applies an argument of [14, Prop. 1.14] (adapted to the discrete setting) to show that (5.1) follows from the Sobolev inequality.

Note that our proof of $(a) \Longrightarrow(b)$ goes through for any $v>0$. If $v>1$, then one could apply the approach of [14] using a Sobolev inequality as an intermediate step between $(a)$ and $(b)$. In general, we use instead a Nash-type inequality that is obtained in the following lemma.

\section{LEMMA 5.2}

Let $(\Gamma, \mu)$ be a weighted graph (which is not necessarily connected). Assume that, for any nonempty finite set $A \subset \Gamma$,

$$
\lambda_{1}(A) \geq \Lambda(\mu(A)),
$$

where $\Lambda(\cdot)$ is a nonnegative nonincreasing function on $(0, \infty)$. Let $f(x)$ be a nonnegative function on $\Gamma$ with finite support. Denote

$$
\sum_{x \in \Gamma} f(x) \mu(x)=a \quad \text { and } \quad \sum_{x \in \Gamma} f^{2}(x) \mu(x)=b .
$$

Then, for any $s>0$,

$$
\frac{1}{2} \sum_{x \sim y}\left(\nabla_{x y} f\right)^{2} \mu_{x y} \geq(b-2 s a) \Lambda(a / s) .
$$

Proof

If $b-2 s a<0$, then (5.3) trivially holds. So, we can assume in the sequel that

$$
s \leq \frac{b}{2 a} .
$$

Since $b \leq a \max f$, (5.4) implies $s<\max f$ and, therefore, the set

$$
A_{s}=\{x \in \Gamma: f(x)>s\}
$$




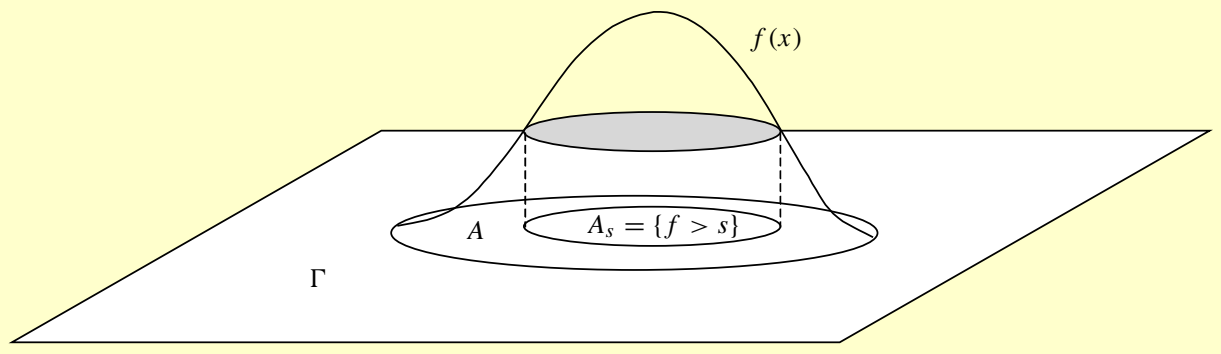

Figure 2. Sets $A$ and $A_{S}$

is nonempty (see Figure 2).

Consider function $h=(f-s)_{+}$. This function belongs to $c_{0}\left(A_{s}\right)$ whence we obtain, by variational property (3.3) of eigenvalues,

$$
\frac{1}{2} \sum_{x \sim y}\left(\nabla_{x y} h\right)^{2} \mu_{x y} \geq \lambda_{1}\left(A_{s}\right) \sum_{x \in \Gamma} h^{2}(x) \mu(x) .
$$

Let us estimate all terms in (5.5) via $f$. We start with the obvious inequality

$$
f^{2} \leq(f-s)_{+}^{2}+2 s f=h^{2}+2 s f
$$

which holds for any $s \geq 0$. It implies $h^{2} \geq f^{2}-2 s f$ whence

$$
\sum_{x \in \Gamma} h^{2}(x) \mu(x) \geq b-2 s a .
$$

The definition of $A_{s}$ implies $\mu\left(A_{s}\right) \leq a / s$ whence, by (5.2),

$$
\lambda_{1}\left(A_{s}\right) \geq \Lambda\left(\mu\left(A_{s}\right)\right) \geq \Lambda(a / s) .
$$

Clearly, we also have

$$
\sum_{x \sim y}\left(\nabla_{x y} h\right)^{2} \mu_{x y} \leq \sum_{x \sim y}\left(\nabla_{x y} f\right)^{2} \mu_{x y} .
$$

Combining this with (5.7), (5.6), and (5.5), we obtain (5.3).

We apply Lemma 5.2 for function $\Lambda(v)=c v^{-1 / v}$. Choosing $s=b /(4 a)$ in (5.3), we obtain

$$
\frac{1}{2} \sum_{x \sim y}\left(\nabla_{x y} f\right)^{2} \mu_{x y} \geq c a^{-2 / v} b^{1+1 / v} .
$$

This is a discrete version of the Nash inequality (cf. [51], [13]). 


\section{Proof of $(a) \Longrightarrow(b)$ in Proposition 5.1}

Step 1. Let $f$ be a nonnegative function on $\Gamma$ with finite support. Denote, for simplicity,

$$
b=\sum_{x \in \Gamma} f^{2}(x) \mu(x) \quad \text { and } \quad b^{\prime}=\sum_{x \in \Gamma}[P f(x)]^{2} \mu(x),
$$

where $P$ is the Markov operator of $(\Gamma, \mu)$. Then we have

$$
b-b^{\prime}=(f, f)_{L^{2}(\Gamma, \mu)}-(P f, P f)_{L^{2}(\Gamma, \mu)}=\left(f,\left(I-P_{2}\right) f\right)_{L^{2}(\Gamma, \mu)} .
$$

Clearly, $Q:=P_{2}$ is also a Markov operator on $\Gamma$ reversible with respect to $\mu$, and it is associated with another structure of a weighted graph on the set $\Gamma$. Denote this weighted graph by $\left(\Gamma^{*}, \mu^{*}\right)$. As a set, $\Gamma^{*}$ coincides with $\Gamma$, and the measures $\mu$ and $\mu^{*}$ on vertices are the same. On the other hand, points $x, y$ are connected by an edge on $\Gamma^{*}$ if there is a path of length 2 from $x$ to $y$ in $\Gamma$, and the weight $\mu_{x y}^{*}$ on edges of $\Gamma^{*}$ is defined by

$$
\mu_{x y}^{*}=Q(x, y) \mu(x) .
$$

Denote by $\Delta^{*}$ the Laplace operator of $\left(\Gamma^{*}, \mu^{*}\right)$. Then $\Delta^{*}=P_{2}-I$ and, by Green formula (3.2),

$$
b-b^{\prime}=-\sum_{x \in \Gamma} f(x) \Delta^{*} f(x) \mu(x)=\frac{1}{2} \sum_{x, y \in \Gamma}\left(\nabla_{x y} f\right)^{2} \mu_{x y}^{*} .
$$

Step 2. If $A$ is a nonempty finite subset of $\Gamma$, then [22, Lem. 4.3] says that*

$$
\lambda_{1}^{*}(A) \geq \lambda_{1}(\bar{A}),
$$

where $\lambda_{1}^{*}(A)$ is the first eigenvalue of $-\Delta_{A}^{*}$. By Faber-Krahn inequality $(F K)$ for the graph $(\Gamma, \mu)$, we obtain

$$
\lambda_{1}^{*}(A) \geq c \mu(\bar{A})^{-1 / v} .
$$

Since $\left(p_{0}\right)$ and Proposition 3.1 imply

$$
\mu(\bar{A}) \leq \sum_{x \in A} V(x, 2) \leq C \sum_{x \in A} \mu(x)=C \mu(A)=C \mu^{*}(A),
$$

(5.11) yields $(F K)$ for the graph $\left(\Gamma^{*}, \mu^{*}\right)$.

\section{Remark 5.1}

The only place where $\left(p_{0}\right)$ is used in the proof of $(a) \Longrightarrow(b)$ is to ensure that $\mu(\bar{A}) \leq C \mu(A)$. If this inequality holds for another reason, then the rest of the proof goes in the same way.

* The proof of (5.10) is based on variational property (3.3) and on the fact that all eigenvalues of $-\Delta_{A}$ belong to the interval $\left[\lambda_{1}(A), 2-\lambda_{1}(A)\right]$. 
Step 3. For some fixed $y \in \Gamma$, denote $f_{n}(x)=p_{n}(x, y)$ and

$$
b_{n}=\sum_{x \in \Gamma} f_{n}^{2}(x) \mu(x)=p_{2 n}(y, y) .
$$

Then $f_{n+1}=P f_{n}$ and we obtain, by (5.9),

$$
b_{n}-b_{n+1}=\frac{1}{2} \sum_{x, y \in \Gamma}\left(\nabla_{x y} f_{n}\right)^{2} \mu_{x y}^{*} .
$$

The graph $\left(\Gamma^{*}, \mu^{*}\right)$ satisfies $(F K)$ so that Lemma 5.2 can be applied. Since

$$
\sum_{x \in \Gamma} f_{n}(x) \mu(x)=\sum_{x \in \Gamma} P_{n}(x, y)=1,
$$

(5.8) yields

$$
\frac{1}{2} \sum_{x, y \in \Gamma}\left(\nabla_{x y} f_{n}\right)^{2} \mu_{x y}^{*} \geq c b_{n}^{1+1 / v}
$$

whence

$$
b_{n}-b_{n+1} \geq c b_{n}^{1+1 / v} .
$$

In particular, we see that $b_{n}>b_{n+1}$.

Next we apply an elementary inequality

$$
v(x-y) \geq \frac{x^{\nu}-y^{\nu}}{x^{\nu-1}+y^{\nu-1}},
$$

which is true for all $x>y>0$ and $v>0$. Taking $x=b_{n+1}^{-1 / v}$ and $y=b_{n}^{-1 / v}$, we obtain, from (5.13) and (5.12),

$v\left(b_{n+1}^{-1 / \nu}-b_{n}^{-1 / v}\right) \geq \frac{b_{n+1}^{-1}-b_{n}^{-1}}{b_{n+1}^{-(\nu-1) / v}+b_{n}^{-(\nu-1) / v}}=\frac{b_{n}-b_{n+1}}{b_{n+1}^{1 / \nu} b_{n}+b_{n}^{1 / \nu} b_{n+1}} \geq \frac{c b_{n}^{1+1 / v}}{2 b_{n}^{1+1 / v}}=\frac{c}{2}$,

whence

$$
b_{n+1}^{-1 / v}-b_{n}^{-1 / v} \geq \frac{c}{2 v}=\text { const. }
$$

Summing up this inequality in $n$, we conclude that $b_{n}^{-1 / v} \geq c n$ and $b_{n} \leq C n^{-v}$.

Since $b_{n}=p_{2 n}(y, y)$, we have proved that, for all $y \in \Gamma$ and $n \geq 1$,

$$
p_{2 n}(y, y) \leq C n^{-v},
$$

which is $(D U E)$ for all even times. 
Step 4. By the semigroup identity, we have, for any $0<k<m$,

$$
p_{m}(x, y)=\sum_{z \in \Gamma} p_{m-k}(x, z) p_{k}(z, y) \mu(z) .
$$

In particular, if $m=2 n, k=n$, and $y=x$, then

$$
p_{2 n}(x, x)=\sum_{z \in \Gamma} p_{n}^{2}(x, z) \mu(z) .
$$

On the other hand, (5.15), the Cauchy-Schwarz inequality, and (5.16) imply

$$
\begin{aligned}
p_{2 n}(x, y) & =\sum_{z \in \Gamma} p_{n}(x, z) p_{n}(z, y) \mu(z) \\
& \leq\left[\sum_{z \in \Gamma} p_{n}^{2}(x, z) \mu(x)\right]^{1 / 2}\left[\sum_{z \in \Gamma} p_{n}^{2}(y, z) \mu(z)\right]^{1 / 2},
\end{aligned}
$$

whence

$$
p_{2 n}(x, y) \leq p_{2 n}(x, x)^{1 / 2} p_{2 n}(y, y)^{1 / 2} .
$$

Together with (5.14), this yields $p_{2 n}(x, y) \leq C n^{-v}$ for all $x, y \in \Gamma$. This implies $(D U E)$ also for odd times if we observe that, by (5.15) and (2.2),

$$
p_{2 n+1}(x, y)=\sum_{z \in \Gamma} p_{2 n}(x, z) P(z, y) \leq \max _{z \in \Gamma} p_{2 n}(x, z) .
$$

\section{Proof of $(c) \Rightarrow($ a) in Proposition 5.1}

Let $A$ be a nonempty finite subset of $\Gamma$, and let $f \in c_{0}(A)$ be the first eigenfunction of $-\Delta_{A}$. We may assume that $f \geq 0$. Let us normalize $f$ so that $\max f=1$, and let $x_{0} \in A$ be the maximum point of $f$. The equation $-\Delta_{A} f=\lambda_{1}(A) f$ implies, by (3.7),

$$
f(x)=\lambda_{1}(A) \sum_{y \in A} G_{A}(x, y) f(y),
$$

whence, for $x=x_{0}$,

$$
1=\lambda_{1}(A) \sum_{y \in A} G_{A}\left(x_{0}, y\right) f(y) \leq \lambda_{1}(A) \sum_{y \in A} G_{A}\left(x_{0}, y\right)
$$

and

$$
\lambda_{1}(A) \geq\left(\max _{x \in A} \sum_{y \in A} G_{A}(x, y)\right)^{-1} .
$$


On the other hand, for any $x \in A$,

$$
\sum_{y \in A} G_{A}(x, y)=\sum_{y \in A} g_{A}(x, y) \mu(y)=\int_{0}^{\infty} \mu\left\{g_{A}(x, \cdot)>t\right\} d t .
$$

Fix some $t_{0}>0$, and estimate the integral above using (5.1), $g_{A} \leq g$, and the fact that

$$
\mu\left\{g_{A}(x, \cdot)>t\right\} \leq \mu(A) .
$$

Then we obtain

$$
\sum_{y \in A} G_{A}(x, y) \leq \int_{0}^{t_{0}} \mu(A) d t+\int_{t_{0}}^{\infty} C t^{-v /(\nu-1)} d t=\mu(A) t_{0}+C t_{0}^{-1 /(\nu-1)} .
$$

Let us choose $t_{0} \simeq \mu(A)^{-(v-1) / v}$ to equate the two terms on the right-hand side, whence

$$
\sum_{y \in A} G_{A}(x, y) \leq C \mu(A)^{1 / \nu} .
$$

Finally, (5.20) and (5.19) imply $(F K)$.

The second result of this section is preceded by two lemmas. We say that a weighted graph $(\Gamma, \mu)$ satisfies the doubling volume condition if

$$
V(x, 2 R) \leq C V(x, R), \quad \forall x \in \Gamma, R>0 .
$$

Clearly, $(D)$ is a weaker assumption than $(V)$.

LEMMA 5.3

If $(\Gamma, \mu)$ satisfies $(D)$, then, for all $x \in \Gamma$ and $R>0$,

$$
\lambda(x, R) \leq \frac{C}{R^{2}} .
$$

\section{Proof}

Let us apply variational property (3.3) with the test function

$$
f(y)=(R-d(x, y))_{+} \in c_{0}(B(x, R)) .
$$

Since $\left|\nabla_{y z} f\right| \leq 1$, (3.3) and (D) imply

$$
\lambda(x, R) \leq \frac{(1 / 2) \sum_{y \sim z}\left(\nabla_{y z} f\right)^{2} \mu_{y z}}{\sum_{y} f^{2}(y) \mu(y)} \leq \frac{C V(x, R)}{R^{2} V(x, R / 2)} \leq \frac{C^{\prime}}{R^{2}},
$$

which was to be proved. 
The next lemma was proved in [59], but we give here a shorter proof.

\section{LEMMA 5.4}

Let $(\Gamma, \mu)$ satisfy $\left(p_{0}\right)$. If $(V)$ and $(G)$ hold, with some positive parameters $\alpha$ and $\gamma$, then $\alpha-\gamma \geq 2$.

\section{Proof}

By (5.19), we have

$$
\lambda(x, R)^{-1} \leq \max _{y \in B(x, R)} \sum_{z \in B(x, 2 R)} G(y, z) .
$$

By $(G)$ and Proposition 3.2, $G(y, y)$ is uniformly bounded from above. Using $(G)$ to estimate $G(y, z)$ for $y \neq z$ and $(V)$, we obtain

$$
\begin{aligned}
\sum_{z \in B(y, 2 R)} G(y, z) & =G(y, y)+\sum_{i=-1}^{\left\lceil\log _{2} R\right\rceil} \sum_{z \in B\left(y, 2^{-i} R\right) \backslash B\left(y, 2^{-i-1} R\right)} g(y, z) \mu(z) \\
& \leq C+C \sum_{i=-1}^{\left\lceil\log _{2} R\right\rceil}\left(2^{-i} R\right)^{-\gamma} V\left(y, 2^{-i} R\right) \\
& \leq C\left[1+\sum_{i=-1}^{\left\lceil\log _{2} R\right\rceil}\left(2^{-i} R\right)^{\alpha-\gamma}\right] .
\end{aligned}
$$

A straightforward computation of sum (5.23) yields, for large $R$,

$$
\sum_{z \in B(y, 2 R)} G(y, z) \leq C \begin{cases}R^{\alpha-\gamma}, & \alpha>\gamma \\ \log _{2} R, & \alpha=\gamma \\ 1, & \alpha<\gamma\end{cases}
$$

Combining (5.22) and (5.24), we obtain

$$
\lambda(x, R) \geq c \begin{cases}R^{-(\alpha-\gamma)}, & \alpha>\gamma, \\ \left(\log _{2} R\right)^{-1}, & \alpha=\gamma, \\ 1, & \alpha<\gamma .\end{cases}
$$

By Lemma 5.3, we have (5.21), which together with (5.25) implies $\alpha-\gamma \geq 2$.

\section{PROPOSITION 5.5}

Let $(\Gamma, \mu)$ satisfy $\left(p_{0}\right)$. If $(V)$ and $(G)$ hold with some positive parameters $\alpha$ and $\gamma$, then Faber-Krahn inequality $(F K)$ holds with the parameter $v=\alpha /(\alpha-\gamma)$. 
Proof

Note that, by Lemma 5.4, we have $\alpha>\gamma$ so that $v$ is positive and, moreover, $v>1$. Let us verify that

$$
\mu\{y: g(x, y)>t\} \leq \text { const } t^{-\alpha / \gamma} .
$$

Then (5.1) would follow with $v=\alpha /(\alpha-\gamma)$, which implies $(F K)$, by Proposition 5.1 .

The upper bound in $(G)$ and $\left(p_{0}\right)$ implies that, for all $x, y$ (including the case $x=y$; see Proposition 3.2),

$$
g(x, y) \leq C \min \left(1, d(x, y)^{-\gamma}\right) .
$$

If $t \geq C$, then the set $\{y: g(x, y)>t\}$ is empty, and (5.26) is trivially true.

Assume now that $t \leq C$. Then (5.27) implies

$$
\mu\{y: g(x, y)>t\} \leq \mu\left\{y: d(x, y)<(t / C)^{-1 / \gamma}\right\}=V\left(x,(t / C)^{-1 / \gamma}\right) .
$$

Since $R:=(t / C)^{-1 / \gamma} \geq 1$, we can apply here the upper bound from $(V)$ and obtain (5.26).

\section{The mean exit time and the Green kernel}

The purpose of this section is to verify part $(V)+(G) \Longrightarrow(E)$ of the proof of Theorem 2.1. Recall that $(E)$ stands for the condition

$$
E(x, R) \simeq R^{\beta}, \quad \forall x \in \Gamma, R \geq 1 .
$$

Alongside the mean exit time $E_{A}(x)$, consider the maximal mean exit time $\bar{E}_{A}$ defined by

$$
\bar{E}_{A}:=\sup _{y} E_{A}(y) \text {. }
$$

If $A=B(x, R)$, then we write $\bar{E}(x, R):=\bar{E}_{B(x, R)}$. We also use the following hypothesis:

$$
\bar{E}(x, R) \leq C E(x, R), \quad \forall x \in \Gamma, R>0 .
$$

PROPOSITION 6.1

The upper bound in (E) implies, for all $x \in \Gamma$ and $R \geq 1$,

$$
\bar{E}(x, R) \leq C R^{\beta} .
$$

The lower bound in (E) implies

$$
\bar{E}(x, R) \geq c R^{\beta} .
$$

Consequently, $(E)$ implies $(\bar{E})$ and

$$
\bar{E}(x, R) \simeq R^{\beta} .
$$




\section{Proof}

To show (6.2), let us observe that, for any point $y \in B(x, R)$, we have $B(x, R) \subset$ $B(y, 2 R)$, whence

$$
\begin{aligned}
\bar{E}(x, R) & =\sup _{y \in B(x, R)} E_{B(x, R)}(y) \leq \sup _{y \in B(x, R)} E_{B(y, 2 R)}(y) \\
& =\sup _{y \in B(x, R)} E(y, 2 R) \leq C R^{\beta} .
\end{aligned}
$$

Lower bound (6.3) is obvious by $E \leq \bar{E}$. Finally, $(\bar{E})$ follows from $(E)$ and (6.4) if $R \geq 1$, and $(\bar{E})$ holds trivially if $R<1$.

PROPOSITION 6.2

For any nonempty finite set $A \subset \Gamma$, we have

$$
\lambda_{1}(A) \geq\left(\bar{E}_{A}\right)^{-1} .
$$

\section{Proof}

Indeed, this is a combination of (5.19) and the definition of $\bar{E}$ (see (3.8) and (6.1)).

The next statement was proved in [59].

\section{PROPOSITION 6.3}

Let $(\Gamma, \mu)$ satisfy $\left(p_{0}\right)$. If $(V)$ and $(G)$ hold, with some positive parameters $\alpha$ and $\gamma$, then $(E)$ holds as well with $\beta=\alpha-\gamma$.

\section{Proof}

Denote $A=B(x, R)$. Applying (3.8), the obvious inequality $g_{A} \leq g$, as well as ( $V$ ) and $(G)$, we obtain (cf. (5.23) and (5.24))

$$
E(x, R)=\sum_{y \in A} g_{A}(x, y) \mu(y) \leq \sum_{y \in A} g(x, y) \mu(y) \leq C R^{\alpha-\gamma} .
$$

Observe that, by Lemma 5.4, we already know that $\alpha>\gamma$.

For the lower bound of $E(x, R)$, let us prove that

$$
g_{A}(x, y) \geq c d(x, y)^{-\gamma}, \quad \forall y \in B(x, \varepsilon R) \backslash\{x\},
$$

provided $\varepsilon>0$ is small enough. Consider the function

$$
u(y)=g(x, y)-g_{A}(x, y)
$$

which is harmonic in $A$. By the maximum principle, its maximum is attained at the boundary of $A$, whence, by $(G)$,

$$
0 \leq u(y) \leq C R^{-\gamma} .
$$


Therefore,

$$
g_{A}(x, y)=g(x, y)-u(y) \geq c d(x, y)^{-\gamma}-C R^{-\gamma} .
$$

If $R$ is large enough and if $d(x, y) \leq \varepsilon R$ with a small enough $\varepsilon$, then the second term in (6.7) is absorbed by the first one, whence (6.6) follows.

Summing up (6.6) over $y$, we obtain (cf. (5.23) and (5.24))

$$
E(x, R)=\sum_{y \in A} g_{A}(x, y) \mu(y) \geq \sum_{y \in B(x, \varepsilon R) \backslash\{x\}} g_{A}(x, y) \mu(y) \geq c R^{\alpha-\gamma} .
$$

If $R$ is not big enough, then the above argument does not work. However, in this case we argue as follows. If the random walk starts at $x$, then $T_{B(x, R)} \geq R$. Hence, we always have $E(x, R)=\mathbb{E}_{x}\left(T_{B(x, R)}\right) \geq R$ which yields the lower bound in $(E)$, provided $R \leq$ const.

Assuming that $(V)$ and $(E)$ hold, there are the following general relations between the exponents $\alpha$ and $\beta$ : if the graph is transient, then $2 \leq \beta \leq \alpha$; and if it is recurrent, then $2 \leq \beta \leq \alpha+1$ (see [59]; see also [60], [61] for various definitions of dimensions of graphs).

\section{Sub-Gaussian term}

The following statement is crucial for obtaining the off-diagonal upper bound of the heat kernel. It contains the part $(E) \Longrightarrow(\Psi)$ of the proof of Theorem 2.1.

\section{PROPOSITION 7.1}

Assume that the graph $(\Gamma, \mu)$ possesses property $(E)$. Then, for all $x \in \Gamma, R>0$, and $n \geq 1$, we have

$$
\Psi_{n}(x, R) \leq C \exp \left(-\left(\frac{R^{\beta}}{C n}\right)^{1 /(\beta-1)}\right) .
$$

We start with the following lemma.

\section{LEMMA 7.2}

Assume that hypothesis $(\bar{E})$ holds on $(\Gamma, \mu)$. Let $A=B\left(x_{0}, r\right)$ be an arbitrary ball on $\Gamma$, and let $v$ be a function on $\bar{A}$ such that $0 \leq v \leq 1$. Suppose that $v$ satisfies in $A$ the equation

$$
\Delta v=\lambda v
$$

where $\lambda$ is a constant such that

$$
\lambda \geq\left(\bar{E}_{A}\right)^{-1}
$$




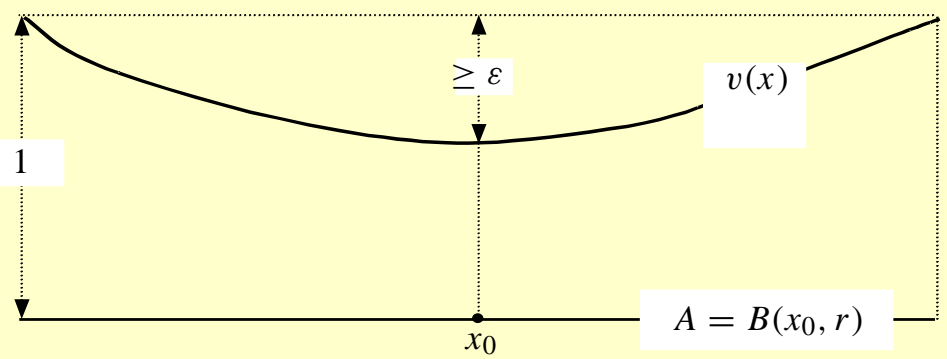

Figure 3. The value of the function $v$ at the point $x_{0}$ does not exceed $1-\varepsilon$

Then

$$
v\left(x_{0}\right) \leq 1-\varepsilon,
$$

where $\varepsilon>0$ depends on the constants in hypothesis $(\bar{E})$ (see Figure 3$)$.

\section{Proof}

Denote for simplicity $u(x)=E_{A}(x)$, and recall that $u \in c_{0}(A)$ and $\Delta u=-1$ in $A$ (cf. (3.9)). Also, denote

$$
\lambda_{0}:=\left(\bar{E}_{A}\right)^{-1}=\frac{1}{\max u} .
$$

Consider the function $w=1-\left(\lambda_{0} / 2\right) u$. Then $1 / 2 \leq w \leq 1$ and, in $A$,

$$
\Delta w=\frac{\lambda_{0}}{2} \leq \lambda_{0} w \leq \lambda w .
$$

Since $v \leq 1$ and $w=1$ outside $A$, the maximum principle for the operator $\Delta-\lambda$ implies that $v \leq w$ in $A$. In particular,

$$
v\left(x_{0}\right) \leq w\left(x_{0}\right)=1-\frac{\lambda_{0}}{2} u\left(x_{0}\right) \leq 1-\frac{u\left(x_{0}\right)}{2 \max u} .
$$

Hypothesis $(\bar{E})$ yields

$$
\frac{u\left(x_{0}\right)}{\max u}=\frac{E\left(x_{0}, r\right)}{\bar{E}\left(x_{0}, r\right)} \geq c,
$$

whence (7.3) follows.

\section{LEMMA 7.3}

Assume that $(\Gamma, \mu)$ satisfies $(E)$. Let $A=B\left(x_{0}, R\right)$ be an arbitrary ball on $\Gamma$, and let $v$ be a function on $\bar{A}$ such that $0 \leq v \leq 1$. If $v$ satisfies, in A, equation (7.1 ) with a constant $\lambda$ such that

$$
C R^{-\beta} \leq \lambda<\bar{\lambda}
$$




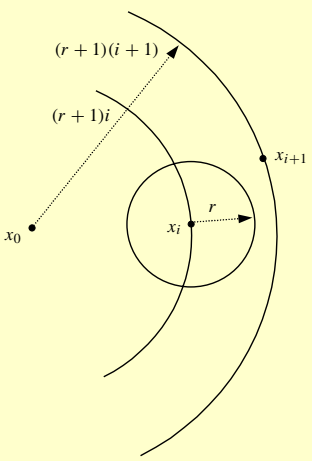

Figure 4. The points $x_{i}$ where $v(x)$ takes the maximum values

then

$$
v\left(x_{0}\right) \leq \exp \left(-c \lambda^{1 / \beta} R\right) .
$$

Here $\bar{\lambda}$ is an arbitrary constant, $C$ is some constant depending on condition $(E)$, and $c>0$ is some constant depending on $\bar{\lambda}$ and on condition $(E)$.

\section{Proof}

Condition $(E)$ implies $(\bar{E})$ and $\bar{E}(x, R) \simeq R^{\beta}$ (see Proposition 6.1). Choose the constant $C$ in (7.4) so big that the lower bound in (7.4) implies $\lambda \geq \bar{E}(x, R)^{-1}$. Then, by Lemma 7.2, we obtain $v\left(x_{0}\right) \leq 1-\varepsilon$. If we have, in addition,

$$
\lambda^{1 / \beta} R \leq \text { const, }
$$

then (7.5) is trivially satisfied. In particular, if $R$ is in the bounded range, then (7.6) is true because $\lambda$ is bounded from above by (7.4).

Hence, we may assume in the sequel that

$$
R>C^{\prime} \quad \text { and } \quad \lambda>C^{\prime \prime} R^{-\beta},
$$

with large enough constants $C^{\prime}$ and $C^{\prime \prime}$ (in particular, $C^{\prime \prime} \gg C$ ). The point of the present lemma is that it improves the previous one for this range of $R$ and $\lambda$. Choose a number $r$ from the equation $\lambda=C r^{-\beta}$, where $C$ is the same constant as in (7.4). The above argument shows that Lemma 7.2 applies in any ball of radius $r$. Let $x_{i}, i \geq 1$, be a point in the ball $B\left(x_{0},(r+1) i\right)$ in which $v$ takes the maximum value in this ball, and denote $m_{i}=v\left(x_{i}\right)$ (see Figure 4). For $i=0$, we set $m_{0}=v\left(x_{0}\right)$.

For each $i \geq 0$, consider the ball $A_{i}=B\left(x_{i}, r\right)$. Since

$$
\bar{A}_{i} \subset B\left(x_{i}, r+1\right) \subset B\left(x_{0},(r+1)(i+1)\right),
$$


we have

$$
\max _{\bar{A}_{i}} v \leq m_{i+1} .
$$

Applying Lemma 7.2 to the function $v / m_{i+1}$ in the ball $A_{i}$, we obtain

$$
m_{i} \leq(1-\varepsilon) m_{i+1}
$$

Iterating this inequality $k:=\lfloor R /(r+1)\rfloor$ times and using $m_{k} \leq 1$, we conclude

$$
v\left(x_{0}\right)=m_{0} \leq(1-\varepsilon)^{k} .
$$

By conditions (7.7) and (7.4) and by the choice of $r$, we have

$$
k \simeq \frac{R}{r} \simeq \lambda^{1 / \beta} R,
$$

so that (7.8) implies (7.5).

\section{LEMMA 7.4}

Assume that $(\Gamma, \mu)$ satisfies $(E)$. Let $A=B\left(x_{0}, R\right)$ be an arbitrary ball on $\Gamma$, and let $w_{n}(x)$ be a function in $\bar{A} \times \mathbb{N}$ such that $0 \leq w \leq 1$. Suppose that $w$ solves in $A \times \mathbb{N}$ the heat equation

$$
w_{n+1}-w_{n}=\Delta w_{n}
$$

with initial data $w_{0} \equiv 0$ in A (see Figure 5). Then, for all $n \geq 1$,

$$
w_{n}\left(x_{0}\right) \leq \exp \left(-c\left(\frac{R^{\beta}}{n}\right)^{1 /(\beta-1)}+1\right) .
$$

Proof

First, consider two trivial cases. If $R^{\beta} \leq C n$, then (7.10) is true just by $w \leq 1$, provided $c$ is small enough. Since $\Delta w(x)$ depends only on the immediate neighbors of $x$, one gets by induction that $w_{k}(x)=0$ for all $x \in B\left(x_{0}, R-k\right)$. Therefore, if $R>n$, then $w_{n}\left(x_{0}\right)=0$, and (7.10) is true again.

Hence, we may assume in the sequel that, for a large enough $C$,

$$
C n^{1 / \beta}<R \leq n
$$

Fix some $\lambda>0$, and find a function $v(x)$ on $\bar{A}$ solving the boundary value problem

$$
\begin{cases}\Delta v=\lambda v & \text { in } A, \\ v=1 & \text { in } \bar{A} \backslash A .\end{cases}
$$




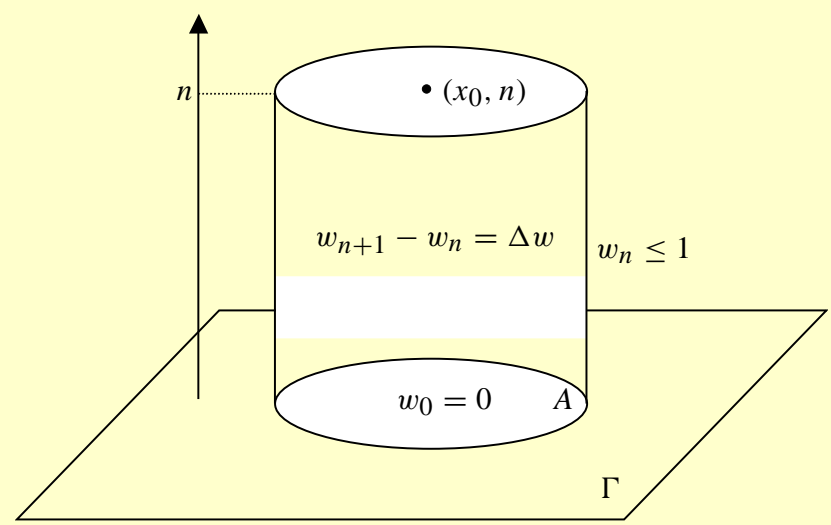

Figure 5. The value of the function $w$ at the point $\left(x_{0}, n\right)$ is affected by the initial value $w=0$ and by the boundary condition

$$
w \leq 1
$$

The function $u_{n}(x):=(1+\lambda)^{n} v(x)$ solves heat equation (7.9) and satisfies the following boundary conditions: $u_{n}(x) \geq 1$ for $x \in \bar{A} \backslash A$ and $u_{0}(x) \geq 0$ for $x \in A$. By the parabolic comparison principle, we have $w \leq u$. Assume for a moment that $\lambda$ satisfies hypothesis (7.4) of Lemma 7.3. Then we estimate $v\left(x_{0}\right)$ by (7.5) and obtain

$$
w_{n}\left(x_{0}\right) \leq(1+\lambda)^{n} v\left(x_{0}\right) \leq \exp \left(\lambda n-c \lambda^{1 / \beta} R\right) .
$$

Now, choose $\lambda$ from the condition $c \lambda^{1 / \beta} R=2 \lambda n$; that is,

$$
\lambda=\left(\frac{c R}{2 n}\right)^{\beta /(\beta-1)} .
$$

As follows from (7.11), this particular $\lambda$ satisfies (7.4). Therefore, the above application of Lemma 7.3 is justified, and we obtain

$$
w_{n}\left(x_{0}\right) \leq \exp (-\lambda n)=\exp \left(-c^{\prime}\left(\frac{R^{\beta}}{n}\right)^{1 /(\beta-1)}\right),
$$

finishing the proof.

\section{Proof of Proposition 7.1}

Denote $A=B\left(x_{0}, R\right)$. By (3.11), the function $w_{n}(x):=\Psi_{n}^{A}(x)$ satisfies all the hypotheses of Lemma 7.4. Hence, $(\Psi)$ follows from (7.10).

\section{Off-diagonal upper bound of the heat kernel}

Here we prove the following implication:

$$
(F K)+(E) \Longrightarrow(U E),
$$


which finishes the proof of the heat kernel upper bound in Theorem 2.1. Indeed, together with the implications

$$
(V)+(G) \stackrel{\text { Prop. } 5.5}{\Longrightarrow}(F K)
$$

and

$$
(V)+(G) \stackrel{\text { Prop. } 6.3}{\Longrightarrow}(E),
$$

(8.1) yields the part $(V)+(G) \Longrightarrow(U E)$ of Theorem 2.1.

PROPOSITION 8.1

On any graph $(\Gamma, \mu)$, we have

$$
(D U E)+(\Psi) \Longrightarrow(U E) .
$$

In particular, if $\left(p_{0}\right)$ holds on $(\Gamma, \mu)$, then

$$
(F K)+(E) \Longrightarrow(U E) .
$$

Proof

By Proposition 5.1, $\left(p_{0}\right)$ and $(F K)$ imply $(D U E)$. By Proposition 7.1, $(E)$ implies $(\Psi)$. Hence, implication (8.3) is a consequence of (8.2).

To prove (8.2), let us fix some points $x, y \in \Gamma$ and denote $r=d(x, y) / 2$. Since balls $B(x, r)$ and $B(y, r)$ do not intersect, the semigroup identity (5.15) and the symmetry of the heat kernel imply, for any triple of nonnegative integers $k, m, n$ such that $k+m=n$,

$$
\begin{aligned}
p_{n}(x, y) & \leq \sum_{z \notin B(x, r)} p_{m}(x, z) p_{k}(z, y) \mu(z)+\sum_{z \notin B(y, r)} p_{m}(x, z) p_{k}(z, y) \mu(z) \\
& \leq \sup _{z} p_{k}(z, y) \sum_{z \notin B(x, r)} P_{m}(x, z)+\sup _{z} p_{m}(x, z) \sum_{z \notin B(y, r)} P_{k}(y, z) \\
& =\sup _{z} p_{k}(y, z) \mathbb{P}_{x}\left(X_{m} \notin B(x, r)\right)+\sup _{z} p_{m}(x, z) \mathbb{P}_{y}\left(X_{k} \notin B(x, r)\right) .
\end{aligned}
$$

As follows from definition (3.10) of $\Psi$,

$$
\mathbb{P}_{x}\left(X_{m} \notin B(x, r)\right) \leq \Psi_{m}(x, r) .
$$

Hence, we obtain the following general inequality, which is true for all reversible random walks:

$$
p_{n}(x, y) \leq \sup _{z} p_{k}(y, z) \Psi_{m}(x, r)+\sup _{z} p_{m}(x, z) \Psi_{k}(y, r) .
$$


As follows from (5.17), diagonal upper bound ( $D U E)$ implies, for all $x, y \in \Gamma$,

$$
p_{n}(x, y) \leq C n^{-\alpha / \beta},
$$

provided $n$ is even. Using inequality (5.18), we see that (8.5) also holds for odd $n$. Assuming $n \geq 2$, choosing $k \simeq m \simeq n / 2$, and applying (8.5) and ( $\Psi$ ) to estimate the right-hand side of (8.4), we obtain ( $U E$ ). If $n=1$, then ( $U E$ ) follows trivially from (8.5) and the fact that $p_{n}(x, y)=0$ whenever $d(x, y)>n$.

\section{On-diagonal lower bound}

In this section we prove part $(\Psi)+(V) \Longrightarrow(D L E)$ of Theorem 2.1.

\section{PROPOSITION 9.1}

Assume that hypothesis $(\Psi)$ holds on $(\Gamma, \mu)$. For arbitrary $x \in \Gamma$ and $R>0$, denote $A=B(x, R)$. Then the following on-diagonal lower bound is true:

$$
p_{2 n}^{A}(x, x) \geq \frac{c}{V\left(x, C n^{1 / \beta}\right)},
$$

provided $n \leq \varepsilon R^{\beta}$, where $\varepsilon$ is a sufficiently small positive constant depending only on the constants from $(\Psi)$.

If in addition $(V)$ holds, then

$$
p_{2 n}^{A}(x, x) \geq c n^{-\alpha / \beta}, \quad \forall n \leq \varepsilon R^{\beta} .
$$

Remark 9.1

Since $p_{2 n} \geq p_{2 n}^{A}$ for any $A=B(x, R)$, inequality $(D L E)$ implies $p_{2 n}(x, x) \geq$ $c n^{-\alpha / \beta}$ for all positive integers $n$.

\section{Proof}

Let us fix some $r \in(0, R)$ and denote $B=B(x, r)$. Since $p^{B} \leq p^{A}$, it suffices to prove (9.1) for $p^{B}$ instead of $p^{A}$, for some $r<R$. Semigroup identity (5.15) for $p^{B}$ and the Cauchy-Schwarz inequality imply

$$
p_{2 n}^{B}(x, x)=\sum_{z \in B} p_{n}^{B}(x, z)^{2} \mu(z) \geq \frac{1}{\mu(B)}\left(\sum_{z \in B} p_{n}^{B}(x, z) \mu(z)\right)^{2} .
$$

Let us observe that

$$
\sum_{z \in B} p_{n}^{B}(\cdot, z) \mu(z)+\Psi_{n}^{B}(\cdot)=1 .
$$

Indeed, the first term in (9.3) is the probability that the random walk $X_{k}$ stays in $B$ up to the time $k=n$, whereas $\Psi_{n}^{B}$ is the probability of the opposite event. 
By hypothesis $(\Psi)$, we have

$$
\Psi_{n}^{B}(x)=\Psi_{n}(x, r) \leq C \exp \left(-\left(\frac{r^{\beta}}{C n}\right)^{1 /(\beta-1)}\right) .
$$

Choosing $r=C n^{1 / \beta}$ for large enough $C$ and assuming $n \leq \varepsilon R^{\beta}$ for sufficiently small $\varepsilon>0$ (the latter ensures $r<R$ ), we obtain, from (9.4), $\Psi_{n}(x, r) \leq 1 / 2$, whence, by (9.3),

$$
\sum_{z \in B} p_{n}^{B}(x, z) \mu(z) \geq \frac{1}{2}
$$

Therefore, (9.2) yields

$$
p_{2 n}^{B}(x, x) \geq \frac{1 / 4}{V(x, r)}=\frac{1 / 4}{V\left(x, C n^{1 / \beta}\right)},
$$

finishing the proof.

\section{The Harnack inequality and the Green kernel}

Recall that the weighted graph $(\Gamma, \mu)$ satisfies the elliptic Harnack inequality if, for all $x \in \Gamma, R>0$, and for any nonnegative function $u$ in $\overline{B(x, 2 R)}$ which is harmonic in $B(x, 2 R)$,

$$
\max _{B(x, R)} u \leq H \min _{B(x, R)} u
$$

with some constant $H>1$. In this section we establish that $(H)$ is implied by condition $(G)$. Recall that the latter refers to

$$
g(x, y) \simeq d(x, y)^{-\gamma}, \quad \forall x \neq y .
$$

Consider the following annulus Harnack inequality for the Green kernel: for all $x \in \Gamma$ and $R>1$,

$$
\max _{y \in A(x, R)} g(x, y) \leq C \min _{y \in A(x, R)} g(x, y),
$$

where $A(x, R):=B(x, R) \backslash B(x, R / 2)$.

PROPOSITION 10.1

Assume that $\left(p_{0}\right)$ hold and the graph $(\Gamma, \mu)$ is transient. Then

$$
(G) \Longrightarrow(H G) \Longrightarrow(H) \text {. }
$$

Since the implication $(G) \Longrightarrow(H G)$ is obvious, we need to prove only the second implication. The main part of the proof is contained in the following lemma. 


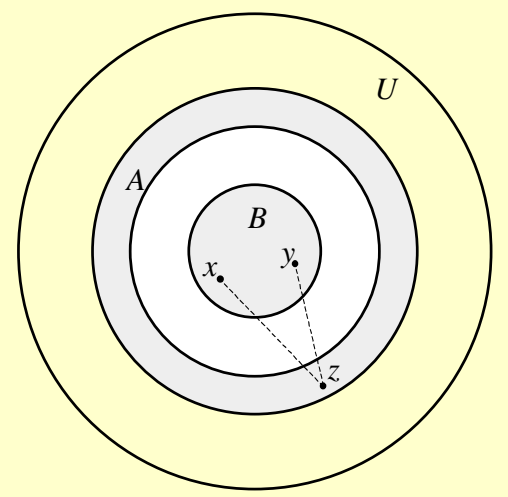

Figure 6. The sets $B=U_{0}, A=U_{2} \backslash U_{1}$, and $U=U_{3}$

LEMMA 10.2

Let $U_{0} \subset U_{1} \subset U_{2} \subset U_{3}$ be a sequence of finite sets in $\Gamma$ such that $\overline{U_{i}} \subset U_{i+1}$, $i=0,1,2$. Denote $A=U_{2} \backslash U_{1}, B=U_{0}$, and $U=U_{3}$. Then, for any function $u$ that is nonnegative in $\overline{U_{2}}$ and harmonic in $U_{2}$, we have

$$
\max _{B} u \leq H \min _{B} u
$$

where

$$
H:=\max _{x \in B} \max _{y \in B} \max _{z \in A} \frac{G_{U}(y, z)}{G_{U}(x, z)}
$$

(see Figure 6).

\section{Remark 10.1}

Note that no a priori assumption has been made about the graph $(\Gamma, \mu)$ (except for connectedness and unboundedness). If the graph is transient, then, by exhausting $\Gamma$ by a sequence of finite sets $U$, we can replace $G_{U}$ in (10.2) by $G$. Note also that, without loss of generality, one can take $U_{2}=\overline{U_{1}}$.

\section{Proof}

The following potential-theoretic argument is borrowed from [12]. We use the notation of Section 3. Given a nonnegative harmonic function $u$ in $U_{2}$, denote by $S_{u}$ the following class of superharmonic functions:

$$
S_{u}=\left\{v: v \geq 0 \text { in } \bar{U}, \Delta v \leq 0 \text { in } U, \text { and } v \geq u \text { in } \overline{U_{1}}\right\} .
$$

Define the function $w$ on $\bar{U}$ by

$$
w(x)=\min \left\{v(x): v \in S_{u}\right\} .
$$




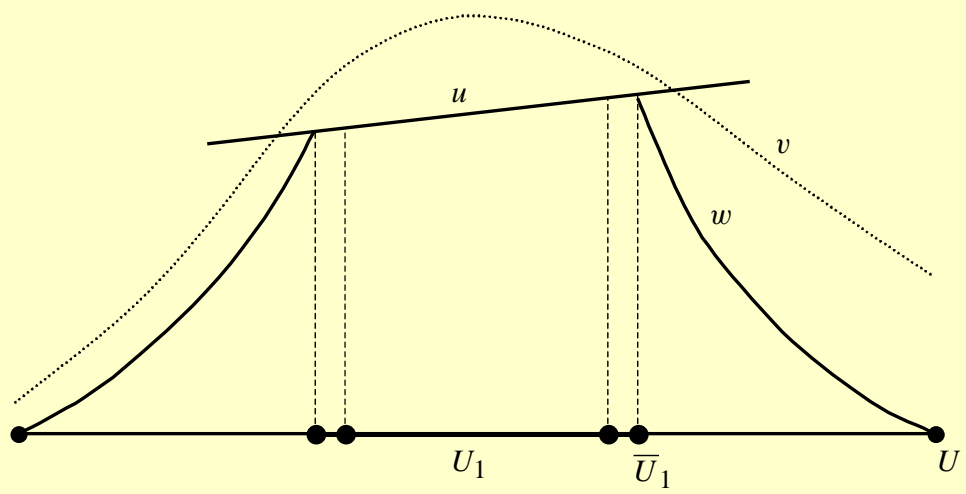

Figure 7. The function $u$, a function $v \in S_{u}$, and the function $w=\min _{S_{u}} v$. The latter is harmonic in $U_{1}$ and in $U \backslash \bar{U}_{1}$.

Clearly, $w \in S_{u}$. Since the function $u$ itself is also in $S_{u}$, we have $w \leq u$ in $\bar{U}$. On the other hand, by definition of $S_{u}, w \geq u$ in $\overline{U_{1}}$, whence we see that $u=w$ in $\overline{U_{1}}$ (see Figure 7). In particular, it suffices to prove (10.1) for $w$ instead of $u$.

Let us show that $w \in c_{0}(U)$. Indeed, let $v(x)=E_{U}(x)$. Then, by (3.9) and the strong minimum principle, $v$ is superharmonic and strictly positive in $U$. Hence, for a large enough constant $C$, we have $C v \geq u$ in $\overline{U_{1}}$, whence $C v \in S_{u}$ and $w \leq C v$. Since $v=0$ in $\bar{U} \backslash U$, this implies $w=0$ in $\bar{U} \backslash U$ and $w \in c_{0}(U)$.

Denote $f:=-\Delta w$, and observe that $f \geq 0$ in $U$. Since $w \in c_{0}(U)$, we have, for any $x \in U$,

$$
w(x)=\sum_{z \in U} G_{U}(x, z) f(z) .
$$

Next we prove that $f=0$ outside $A$ so that the summation in (10.4) can be restricted to $z \in A$. Given this, we obtain, for all $x, y \in B$,

$$
\frac{w(y)}{w(x)}=\frac{\sum_{z \in A} G_{U}(y, z) f(z)}{\sum_{z \in A} G_{U}(x, z) f(z)} \leq H,
$$

whence (10.1) follows.

We are left to verify that $w$ is harmonic in $U_{1}$ and outside $\overline{U_{1}}$. Indeed, if $x \in U_{1}$, then

$$
\Delta w(x)=\Delta u(x)=0
$$

because $w=u$ in $\overline{U_{1}}$. Let $\Delta w(x) \neq 0$ for some $x \in U \backslash \overline{U_{1}}$. Since $w$ is superharmonic, we have $\Delta w(x)<0$ and

$$
w(x)>P w(x)=\sum_{y \sim x} P(x, y) w(y) .
$$


Consider the function $w^{\prime}$, which is equal to $w$ everywhere in $\bar{U}$ except for the point $x$; and $w^{\prime}$ at $x$ is defined to satisfy

$$
w^{\prime}(x)=\sum_{y \sim x} P(x, y) w^{\prime}(y) .
$$

Clearly, $w^{\prime}(x)<w(x)$, and $w^{\prime}$ is superharmonic in $U$. Since $w^{\prime}=w=u$ in $\overline{U_{1}}$, we have $w^{\prime} \in S_{u}$. Hence, by definition (10.3) of $w, w \leq w^{\prime}$ in $\bar{U}$, which contradicts $w(x)>w^{\prime}(x)$.

\section{Proof of Proposition 10.1}

Now we assume $(H G)$ and prove $(H)$. Given any ball $B\left(x_{0}, 2 R\right)$ of radius $R>4$ and a nonnegative harmonic function $u$ in $B\left(x_{0}, 2 R\right)$, define the sequence of radii $R_{0}=R, R_{1}=3 R / 2$, and $R_{2}=2 R$, and denote $U_{i}=B\left(x_{0}, R_{i}\right)$ for $i=0,1,2$ and $U_{3}=\Gamma$. By Lemma 10.2, we have inequality (10.1), which implies $(H)$, provided we can show that the Harnack constant $H$ from (10.2) is bounded from above, uniformly in $x_{0}$ and $R$. Indeed, if $x, y \in B\left(x_{0}, R\right)$ and $z \in A=\overline{B\left(x_{0}, 2 R\right)} \backslash B\left(x_{0}, 3 R / 2\right)$, then both distances $d(z, x)$ and $d(z, y)$ are between $R / 2$ and 7R/2. By iterating $(H G)$ in the annuli centered at $z$, we obtain

$$
\frac{G(y, z)}{G(x, z)}=\frac{g(z, y)}{g(z, x)} \leq \mathrm{const},
$$

whence we see that $H$ is indeed uniformly bounded from above.

The condition $R>4$, which we have imposed above, ensures that $\overline{U_{i}} \subset U_{i+1}$, which is required for Lemma 10.2. If $R \leq 4$, then $(H)$ simply follows from $\left(p_{0}\right)$ and Proposition 3.2.

\section{Oscillation inequalities}

For any nonempty finite set $U$ and a function $u$ on $U$, denote

$$
\underset{U}{\operatorname{osc}} u:=\max _{U} u-\min _{U} u .
$$

The purpose of this section is to prove estimate (11.3), which provides the step $(H) \Longrightarrow[\mathrm{osc}]$ of the proof of Theorem 2.1.

PROPOSITION 11.1

Assume that elliptic Harnack inequality $(H)$ holds on $(\Gamma, \mu)$. Then, for any $\varepsilon>0$, there exists $\sigma=\sigma(\varepsilon, H)<1$ such that, for any ball $B(x, R)$ and for any function $u$ defined in $\overline{B(x, R)}$ and harmonic in $B(x, R)$, we have

$$
\underset{B(x, \sigma R)}{\operatorname{osc}} u \leq \varepsilon \underset{B(x, R)}{\operatorname{osc}} u .
$$




\section{Proof}

Fix a ball $B(x, R)$, and denote for simplicity $B_{r}=B(x, r)$. Let us prove that, for any $r \in(0, R / 3]$,

$$
\underset{B_{r}}{\operatorname{osc}} u \leq(1-\delta) \underset{B_{3 r}}{\operatorname{osc}} u,
$$

where $\delta=\delta(H) \in(0,1)$. Then (11.1) follows from (11.2) by iterating.

If $r \leq 1$, then the left-hand side of (11.2) vanishes and (11.2) is trivially satisfied. If $r>1$, then $\overline{B_{2 r}} \subset B_{3 r}$, and the function $u-\min _{B_{3 r}} u$ is nonnegative in $\overline{B_{2 r}}$ and harmonic in $B_{2 r}$. Applying Harnack inequality $(H)$ to this function, we obtain

$$
\max _{B_{r}} u-\min _{B_{3 r}} u \leq H\left(\min _{B_{r}} u-\min _{B_{3 r}} u\right)
$$

and

$$
\underset{B_{r}}{\operatorname{osc}} u \leq(H-1)\left(\min _{B_{r}} u-\min _{B_{3 r}} u\right) .
$$

Similarly, we have

$$
\underset{B_{r}}{\operatorname{osc}} u \leq(H-1)\left(\max _{B_{3 r}} u-\max _{B_{r}} u\right) .
$$

Summing up these two inequalities, we conclude

$$
\underset{B_{r}}{\operatorname{osc}} u \leq C\left(\underset{B_{3 r}}{\operatorname{osc}} u-\underset{B_{r}}{\operatorname{osc}} u\right),
$$

whence (11.2) follows.

PROPOSITION 11.2

Assume that elliptic Harnack inequality $(H)$ holds on $(\Gamma, \mu)$. Let $u \in c_{0}(B(x, R))$ satisfy in $B(x, R)$ the equation $\Delta u=f$. Then, for any positive $r<R$,

$$
\underset{B(x, \sigma r)}{\operatorname{osc}} u \leq 2(\bar{E}(x, r)+\varepsilon \bar{E}(x, R)) \max |f|,
$$

where $\sigma$ and $\varepsilon$ are the same as in Proposition 11.1.

Proof

Denote for simplicity $B_{r}=B(x, r)$. By definition of the Green function, we have

$$
u(y)=-\sum_{z \in B_{R}} G_{B_{R}}(y, z) f(z),
$$

whence, using (3.8), we obtain

$$
\max |u| \leq \bar{E}(x, R) \max |f| .
$$




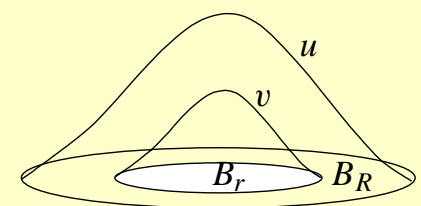

Figure 8. The functions $u$ and $v$ in the case $f \leq 0$

Let $v \in c_{0}\left(B_{r}\right)$ solve the Dirichlet problem $\Delta v=f$ in $B_{r}$ (see Figure 8). In the same way, we have

$$
\max |v| \leq \bar{E}(x, r) \max |f| .
$$

The function $w=u-v$ is harmonic in $B_{r}$ whence, by Proposition 11.1,

$$
\underset{B_{\sigma r}}{\operatorname{osc}} w \leq \varepsilon \underset{B_{r}}{\operatorname{osc}} w .
$$

Since $w=u$ on $\overline{B_{r}} \backslash B_{r}$, the maximum principle implies that

$$
\underset{B_{r}}{\operatorname{osc}} w=\underset{B_{r} \backslash B_{r}}{\operatorname{osc}} w=\frac{\operatorname{osc}}{B_{r} \backslash B_{r}} u \leq 2 \max |u| .
$$

Hence,

$\underset{B_{\sigma r}}{\operatorname{osc}} u \leq \underset{B_{\sigma r}}{\operatorname{osc}} v+\underset{B_{\sigma r}}{\operatorname{osc}} w \leq 2 \max |v|+2 \varepsilon \max |u| \leq 2(\bar{E}(x, r)+\varepsilon \bar{E}(x, R)) \max |f|$, which was to be proved.

\section{Time derivative of the heat kernel}

Given a function $u_{n}(x)$ on $\Gamma \times \mathbb{N}$, by the "time derivative" of $u$ we mean the difference

$$
\partial_{n} u:=u_{n+2}-u_{n} .
$$

The main result of this section is Proposition 12.3, which provides upper bound (12.5) for $\partial_{n} p$ and thus constitutes the part $(D U E) \Longrightarrow$ [deriv] of the proof of Theorem 2.1. The crucial point is that $\partial_{n} p$ decays as $n \rightarrow \infty$ faster than $p_{n}$.

The analogue of the time derivative in the discrete case is $\partial_{n} p=p_{n+2}-p_{n}$ rather than $p_{n+1}-p_{n}$. Indeed, in $\mathbb{Z}^{D}$ (as well as in any other bipartite graph), $p_{n}(x, x)=0$ if $n$ is odd. Therefore, the difference $p_{n+1}(x, x)-p_{n}(x, x)$ is equal either to $p_{n+1}(x, x)$ or to $-p_{n}(x, x)$, and hence it decays as $n \rightarrow \infty$ at the same rate as $p_{n}(x, x)$.

\section{PROPOSITION 12.1}

Let $A$ be a nonempty finite subset of $\Gamma$, and let $f$ be a function on $A$. Define

$$
u_{n}(x)=P_{n}^{A} f(x) .
$$


Then, for all integers $1 \leq k \leq n$,

$$
\left\|\partial_{n} u\right\|_{L^{2}(A, \mu)} \leq \frac{1}{k}\left\|u_{n-k}\right\|_{L^{2}(A, \mu)} .
$$

Proof

The proof follows the argument from [17]. Let $\phi_{1}, \phi_{2}, \ldots, \phi_{|A|}$ be the eigenfunctions of the Laplace operator $-\Delta_{A}$, and let $\lambda_{1}, \lambda_{2}, \ldots, \lambda_{|A|}$ be the corresponding eigenvalues. Let us normalize $\phi_{i}$ 's to form an orthonormal basis in $L^{2}(A, \mu)$. The function $f$ can be expanded in this basis:

$$
f=\sum_{i} c_{i} \phi_{i}
$$

Since $P^{A}=I-\left(-\Delta_{A}\right)$, we obtain

$$
u_{n}=\sum_{i} \rho_{i}^{n} \phi_{i},
$$

where $\rho_{i}:=1-\lambda_{i}$ are eigenvalues of the Markov operator $P^{A}$.

From (12.1), we obtain

$$
u_{n}-u_{n+2}=\sum\left(1-\rho_{i}^{2}\right) \rho_{i}^{n} \phi_{i}
$$

and

$$
\left\|u_{n}-u_{n+2}\right\|_{L^{2}(A, \mu)}^{2}=\sum_{i}\left(1-\rho_{i}^{2}\right)^{2} \rho_{i}^{2 n} .
$$

Note that $\left|\rho_{i}\right| \leq 1$ and hence $\rho_{i}^{2} \in[0,1]$. For any $a \in[0,1]$, we have

$$
1 \geq\left(1+a+a^{2}+\cdots+a^{k}\right)(1-a) \geq k a^{k}(1-a),
$$

whence

$$
(1-a) a^{k} \leq \frac{1}{k}
$$

Applying this inequality for $a=\rho_{i}^{2}$, we obtain, from (12.2),

$$
\left\|u_{n}-u_{n+2}\right\|_{L^{2}(A, \mu)}^{2} \leq \frac{1}{k^{2}} \sum_{i} \rho_{i}^{2(n-k)}=\frac{1}{k^{2}}\left\|u_{n-k}\right\|_{L^{2}(A, \mu)}^{2},
$$

which was to be proved.

PROPOSITION 12.2

Let $A$ be a nonempty finite subset of $\Gamma$. Then, for all $x, y \in A$,

$$
\left|\partial_{n} p^{A}(x, y)\right| \leq \frac{1}{k} \sqrt{p_{2 m}^{A}(x, x) p_{2(n-m-k)}^{A}(y, y)}
$$

for all positive integers $n, m, k$ such that $m+k \leq n$. 


\section{Proof}

From semigroup identity (5.15) for $p^{A}$, we obtain

$$
\partial_{n} p^{A}(x, y)=\sum_{z \in A} p_{m}^{A}(x, z) \partial_{n-m} p^{A}(z, y) \mu(z),
$$

whence

$$
\left|\partial_{n} p^{A}(x, y)\right| \leq\left\|p_{m}^{A}(x, \cdot)\right\|_{L^{2}(A, \mu)}\left\|\partial_{n-m} p^{A}(y, \cdot)\right\|_{L^{2}(A, \mu)} .
$$

By Proposition 12.1,

$$
\left\|\partial_{n-m} p^{A}(y, \cdot)\right\|_{L^{2}(A, \mu)} \leq \frac{1}{k}\left\|p_{n-m-k}^{A}(y, \cdot)\right\|_{L^{2}(A, \mu)}
$$

for any $1 \leq k \leq n-m$. Since

$$
\left\|p_{m}^{A}(x, \cdot)\right\|_{L^{2}(A, \mu)}^{2}=\sum_{z \in A} p_{m}^{A}(x, z)^{2} \mu(z)=p_{2 m}^{A}(x, x),
$$

we obtain (12.3).

PROPOSITION 12.3

Suppose that (DUE) holds; that is, for all $x \in \Gamma$ and $n \geq 1$,

$$
p_{n}(x, x) \leq C n^{-v}
$$

Then, for all $x, y \in \Gamma$ and $n \geq 1$,

$$
\left|\partial_{n} p(x, y)\right| \leq C n^{-v-1}
$$

\section{Proof}

First, assume $n>3$. Then we can choose $k$ and $m$ in (12.3) so that $k \simeq m \simeq n / 3$ and $n-m-k \simeq n / 3$. As follows from (12.4), for any nonempty finite set $A \subset \Gamma$,

$$
p_{2 m}^{A}(x, x) \leq C n^{-v} \quad \text { and } \quad p_{2(n-m-k)}^{A}(y, y) \leq C n^{-v},
$$

whence, by Proposition 12.1,

$$
\left|\partial_{n} p^{A}(x, y)\right| \leq C n^{-v-1}
$$

By letting $A \rightarrow \Gamma$, we obtain (12.5).

If $n \leq 3$, then (12.5) follows from the trivial inequality $\left|\partial_{n} p\right| \leq p_{n}+p_{n+2}$ and the fact that (12.4) implies a similar bound for $p_{n}(x, y)$ (cf. (5.17) and (5.18)). 


\section{Off-diagonal lower bound}

An important intermediate step in proving the lower estimate $(L E)$ is a near-diagonal lower estimate

$$
p_{n}(x, y)+p_{n+1}(x, y) \geq c n^{-\alpha / \beta}
$$

for all $x, y \in \Gamma$ and $n \geq 1$ such that

$$
d(x, y) \leq \delta n^{1 / \beta} .
$$

In this section we finish the proof of lower bound $(L E)$ in Theorem 2.1 as on the following diagram:

$$
(V)+(G) \Longrightarrow(F K)+(V)+(E)+(H) \Longrightarrow(N L E)+(V) \Longrightarrow(L E) .
$$

The first implication here is given by Propositions 5.5, 6.3, and 10.1, whereas the other two are proved below.

Let us recall that $(D L E)$ refers to the lower bound

$$
p_{2 n}^{B(x, R)}(x, x) \geq c n^{-\alpha / \beta}, \quad \forall n \leq \varepsilon R^{\beta},
$$

with some small enough $\varepsilon>0$, and $(D U E)$ refers to the upper bound

$$
p_{n}(x, x) \leq C n^{-\alpha / \beta} .
$$

Denote for simplicity by $(E \leq)$ the upper bound in $(E)$; that is,

$$
E(x, R) \leq C R^{\beta}, \quad \forall x \in \Gamma, R \geq 1 .
$$

PROPOSITION 13.1

For any graph $(\Gamma, \mu)$, we have

$$
(D U E)+(D L E)+(E \leq)+(H) \Longrightarrow(N L E) .
$$

Consequently, if $\left(p_{0}\right)$ holds on $(\Gamma, \mu)$, then

$$
(F K)+(V)+(E)+(H) \Longrightarrow(N L E) .
$$

\section{Proof}

Let us first show how the second claim follows from the first one. Recall that, by Proposition 5.1, $(F K) \Longrightarrow(D U E)$; by Proposition 7.1, $(E) \Longrightarrow(\Psi)$; and, by Proposition 9.1, $(\Psi)+(V) \Longrightarrow(D L E)$. Hence, the hypotheses of (13.3) imply the hypotheses of (13.2).

To prove (13.2), fix $x \in \Gamma, n \geq 1$, and set

$$
R=\left(\frac{n}{\varepsilon}\right)^{1 / \beta}
$$


for a small enough positive $\varepsilon$. So far we only assume that $\varepsilon$ satisfies $(D L E)$, but later one more upper bound on $\varepsilon$ is imposed. Denote $A=B(x, R)$, and introduce the function

$$
u(y):=p_{n}^{A}(x, y)+p_{n+1}^{A}(x, y) .
$$

By hypothesis $(D L E)$, we have $u(x) \geq c n^{-\alpha / \beta}$. Let us show that

$$
|u(x)-u(y)| \leq \frac{c}{2} n^{-\alpha / \beta}
$$

for all $y$ such that $d(x, y) \leq \delta n^{1 / \beta}$, which would imply $u(y) \geq(c / 2) n^{-\alpha / \beta}$ and hence prove $(N L E)$.

The function $u(y)$ is in the class $c_{0}(A)$ and solves the equation $\Delta u(y)=f(y)$ where

$$
f(y):=p_{n+2}^{A}(x, y)-p_{n}^{A}(x, y) .
$$

On-diagonal upper bound ( $D U E$ ) implies, by Proposition 12.3,

$$
\max _{y}|f(y)| \leq \frac{C}{n^{\alpha / \beta+1}} .
$$

By $(H)$ and Proposition 11.2, we have, for any $0<r<R$ and for some $\sigma \in(0,1)$,

$$
\underset{B(x, \sigma r)}{\operatorname{osc}} u \leq 2\left(\bar{E}(x, r)+\varepsilon^{2} \bar{E}(x, R)\right) \max |f| .
$$

By Proposition 6.1, $(E \leq)$ implies a similar upper bound for $\bar{E}$. Estimating $\max |f|$ by (13.6), we obtain, from (13.7),

$$
\underset{B(x, \sigma r)}{\operatorname{osc}} u \leq C \frac{r^{\beta}+\varepsilon^{2} R^{\beta}}{n^{\alpha / \beta+1}} .
$$

Choosing $r$ to satisfy $r^{\beta}=\varepsilon^{2} R^{\beta}$ and substituting from (13.4) $n=\varepsilon R^{\beta}$, we obtain

$$
\underset{B(x, \sigma r)}{\operatorname{osc}} u \leq C \frac{\varepsilon^{2} R^{\beta}}{n^{\alpha / \beta+1}}=C \varepsilon n^{-\alpha / \beta},
$$

which implies

$$
\underset{B(x, \sigma r)}{\operatorname{osc}} u \leq \frac{c}{2} n^{-\alpha / \beta}
$$

provided $\varepsilon$ is small enough.

Note that

$$
\sigma r=\sigma \varepsilon^{2 / \beta} R=\sigma \varepsilon^{2 / \beta}\left(\frac{n}{\varepsilon}\right)^{1 / \beta}=\sigma \varepsilon^{1 / \beta} n^{1 / \beta}=\delta n^{1 / \beta},
$$

where $\delta:=\sigma \varepsilon^{1 / \beta}$. Hence, (13.8) implies (13.5), provided $d(x, y) \leq \delta n^{1 / \beta}$, which was to be proved. 
The final step in proving part $(V)+(G) \Longrightarrow(L E)$ of Theorem 2.1 is covered by the following statement. Denote by $(V \geq)$ the lower bound in $(V)$ that is,

$$
V(x, R) \geq c R^{\alpha}, \quad \forall x \in \Gamma, R \geq 1 .
$$

PROPOSITION 13.2

Assume that $(\Gamma, \mu)$ satisfies $\left(p_{0}\right)$. Then

$$
(N L E)+(V \geq) \Longrightarrow(L E) .
$$

We precede the proof with the following lemmas. Denote for simplicity

$$
\tilde{P}_{n}=P_{n}+P_{n+1},
$$

where $P_{n}$ is the $n$-convolution power of the Markov operator $P$. In particular, we have

$$
P_{n} P_{m}=P_{n+m} .
$$

We need a replacement for this property for the operator $\tilde{P}_{n}$, which is stated in Lemma 13.5 .

LEMMA 13.3

Assume that $\left(p_{0}\right)$ holds on $(\Gamma, \mu)$. Then, for all integers $n \geq l \geq 1$ such that

$$
n \equiv l \quad(\bmod 2),
$$

we have

$$
P_{l}(x, y) \leq C^{n-l} P_{n}(x, y)
$$

for all $x, y \in \Gamma$, with a constant $C=C\left(p_{0}\right)$.

\section{Proof}

By semigroup property (5.15), we have

$$
P_{k+2}(x, y)=\sum_{z \in \Gamma} P_{k}(x, z) P_{2}(z, y) \geq P_{k}(x, y) P_{2}(y, y) .
$$

Using $\left(p_{0}\right)$, we obtain

$$
P_{2}(y, y)=\sum_{z \sim y} P(y, z) P(z, y) \geq p_{0} \sum_{z \sim y} P(y, z)=p_{0},
$$

whence $P_{k+2}(x, y) \geq p_{0} P_{k}(x, y)$. Iterating this inequality, we obtain (13.13) with $C=p_{0}^{-1 / 2}$. 
LEMMA 13.4

Assume that $(\Gamma, \mu)$ satisfies $\left(p_{0}\right)$. Then, for all integers $n \geq l \geq 1$ and all $x, y \in \Gamma$,

$$
\tilde{P}_{l}(x, y) \leq C^{n-l} \tilde{P}_{n}(x, y),
$$

where $C=C\left(p_{0}\right)$.

\section{Remark 13.1}

Note that no parity condition is required here in contrast to condition (13.12) of Lemma 13.3.

\section{Proof}

This is an immediate consequence of Lemma 13.3 because both $P_{l}(x, y)$ and $P_{l+1}(x, y)$ can be estimated from above via either $P_{n}(x, y)$ or $P_{n+1}(x, y)$ depending on the parity of $n$ and $l$.

\section{LEMMA 13.5}

Assume that $(\Gamma, \mu)$ satisfies $\left(p_{0}\right)$. Then, for all $n, m \in \mathbb{N}$ and $x, y \in \Gamma$, we have the following inequality:

$$
\tilde{P}_{n} \tilde{P}_{m}(x, y) \leq C \tilde{P}_{n+m+1}(x, y),
$$

where $C=C\left(p_{0}\right)$.

\section{Proof}

Observe that, by (13.10) and (13.11),

$$
\tilde{P}_{n} \tilde{P}_{m}=\left(P_{n}+P_{n+1}\right)\left(P_{m}+P_{m+1}\right)=P_{n+m}+2 P_{n+m+1}+P_{n+m+2} .
$$

By Lemma 13.3, $P_{n+m}(x, y) \leq C P_{n+m+2}$, whence

$$
\tilde{P}_{n} \tilde{P}_{m}(x, y) \leq C\left(P_{n+m+1}+P_{n+m+2}\right)=C \tilde{P}_{n+m+1} .
$$

\section{LEMMA 13.6}

Assume that $(\Gamma, \mu)$ satisfies $\left(p_{0}\right)$. Then, for all $x, y \in \Gamma$ and $k, m, n \in \mathbb{N}$ such that $n \geq k m+k-1$, we have the following inequality:

$$
\left(\tilde{P}_{m}\right)^{k}(x, y) \leq C^{n-k m} \tilde{P}_{n}(x, y) .
$$

\section{Proof}

By induction, (13.15) implies

$$
\left(\tilde{P}_{m}\right)^{k}(x, y) \leq C^{k-1} \tilde{P}_{k m+k-1}(x, y) .
$$


From inequality (13.14) with $l=k m+k-1$, we obtain

$$
\tilde{P}_{k m+k-1}(x, y) \leq C^{n-k m-(k-1)} \tilde{P}_{n}(x, y),
$$

whence (13.16) follows.

\section{Proof of Proposition 13.2}

Since

$$
\tilde{P}_{n}(x, y)=\left(p_{n}(x, y)+p_{n+1}(x, y)\right) \mu(y),
$$

$(N L E)$ can be stated as follows:

$$
\tilde{P}_{n}(x, y) \geq c n^{-\alpha / \beta} \mu(y) \text { if } d(x, y) \leq \delta n^{1 / \beta} .
$$

The required $(L E)$ takes the form

$$
\tilde{P}_{n}(x, y) \geq c n^{-\alpha / \beta} \mu(y) \exp \left[-\left(\frac{d^{\beta}(x, y)}{c n}\right)^{1 /(\beta-1)}\right] .
$$

To prove (13.18), fix $x, y \in \Gamma, n \geq d(x, y)$, and consider the following cases:

Case 1: $d(x, y) \leq \delta n^{1 / \beta}$,

Case 2: $\delta n^{1 / \beta}<d(x, y) \leq \varepsilon n$,

Case 3: $\varepsilon n<d(x, y) \leq n$.

Here $\delta$ is the constant from (13.17) and $\varepsilon>0$ is a small constant to be chosen later. In the first case, (13.18) coincides with (13.17). In the third case, (13.18) becomes

$$
\tilde{P}_{n}(x, y) \geq c n^{-\alpha / \beta} \mu(y) \exp (-C n),
$$

which can be deduced directly from $\left(p_{0}\right)$. Indeed, depending on the parity of $n$, there is a path from $x$ to $y$ of length either $n$ or $n+1$. The $\mathbb{P}_{x}$-probability that the random walk follows this path is at least $p_{0}^{-(n+1)}$, whence

$$
\tilde{P}_{n}(x, y) \geq \exp (-C n) .
$$

This implies (13.19), using the fact that $\mu(y) \leq C$. The latter is proved as follows. Take, in (13.17), $x \sim y$ and $n \simeq \delta^{-\beta}$. Then (13.17) implies

$$
1 \geq \tilde{P}_{n}(x, y) \geq c \delta^{\alpha} \mu(y),
$$

whence $\mu(y) \leq C$.

Consider the main second case. Denote $d=d(x, y)$, take a positive integer $k$ such that

$$
k \leq d
$$



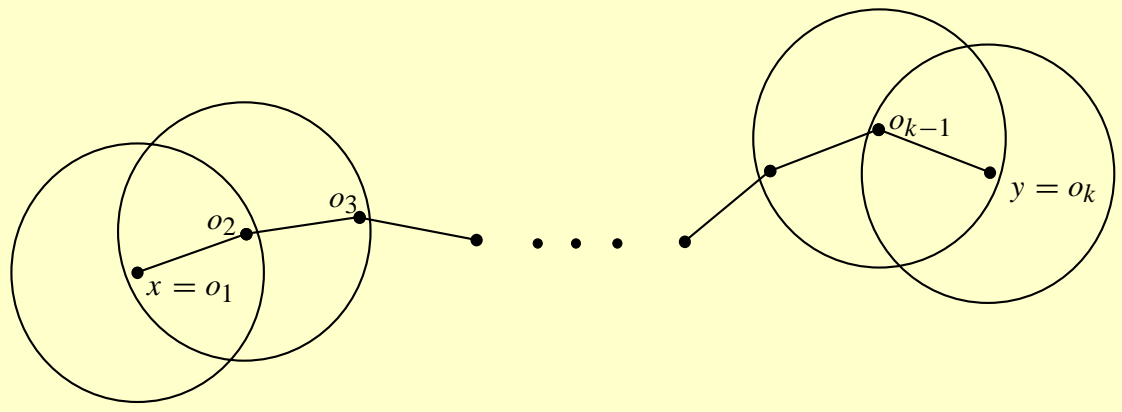

Figure 9. The chain of balls $B\left(o_{i}, r\right)$

and define $m$ by

$$
m=\left\lfloor\frac{n}{k}\right\rfloor-1 .
$$

Since $k \leq d \leq \varepsilon n$, we see that $n / k \geq \varepsilon^{-1}$ and that $m$ is positive. Since $n \geq k(m+1)$, Lemma 13.6 applies and yields

$$
C^{n-m k} \tilde{P}_{n}(x, y) \geq\left(\tilde{P}_{m}\right)^{k}(x, y) .
$$

In order to estimate $\left(\tilde{P}_{m}\right)^{k}(x, y)$, observe that there exists a sequence $o_{1}, o_{2}, \ldots, o_{k}$ of points on $\Gamma$ such that $x=o_{1}, y=o_{k}$, and, for all $i=1,2, \ldots, k-1$,

$$
d\left(o_{i}, o_{i+1}\right) \leq\left\lceil\frac{d(x, y)}{k}\right\rceil=: r
$$

(see Figure 9).

Clearly, we have

$$
\left(\tilde{P}_{m}\right)^{k}(x, y) \geq \sum_{z_{1} \in B\left(o_{1}, r\right)} \cdots \sum_{z_{k-1} \in B\left(o_{k-1}, r\right)} \tilde{P}_{m}\left(x, z_{1}\right) \tilde{P}_{m}\left(z_{1}, z_{2}\right) \cdots \tilde{P}_{m}\left(z_{k-1}, y\right) .
$$

Assume that we have, in addition,

$$
3 r \leq \delta m^{1 / \beta} .
$$

Since $d\left(z_{i-1}, z_{i}\right) \leq 3 r$, each $\tilde{P}_{m}\left(z_{i-1}, z_{i}\right)$ can be estimated by (13.17) as follows:

$$
\tilde{P}_{m}\left(z_{i-1}, z_{i}\right) \geq c m^{-\alpha / \beta} \mu\left(z_{i}\right) .
$$

The same applies to $\tilde{P}_{m}\left(x, z_{1}\right)$ and $\tilde{P}_{m}\left(z_{k-1}, y\right)$. Using the lower bound of volume (13.9), we obtain, from (13.22) and 13.24),

$$
\begin{aligned}
C^{n-m k} \tilde{P}_{n}(x, y) & \geq\left(c m^{-\alpha / \beta}\right)^{k-1} V\left(o_{1}, r\right) \cdots V\left(o_{k-1}, r\right) \mu(y) \\
& \geq c^{k} m^{-(\alpha / \beta) k} r^{\alpha(k-1)} \mu(y) .
\end{aligned}
$$


Hence,

$$
\tilde{P}_{n}(x, y) \geq c^{n-m k+k} m^{-(\alpha / \beta) k} r^{\alpha(k-1)} \geq c^{k} m^{-\alpha / \beta}\left(\frac{r}{m^{1 / \beta}}\right)^{\alpha(k-1)},
$$

where we have used the fact that $n-m k+k \leq 3 k$, which follows from (13.21).

Before we go further, let us specify the choice of $k$ to ensure that both (13.20) and (13.25) hold. Using definitions (13.21) and (13.23) of $m$ and $r$, we see that (13.25) is equivalent to

$$
C \frac{d}{k} \leq \delta\left(\frac{n}{k}\right)^{1 / \beta}
$$

or

$$
k \geq C \delta^{-\beta /(\beta-1)}\left(\frac{d^{\beta}}{n}\right)^{1 /(\beta-1)} .
$$

Let $k$ be the minimal possible integer satisfying (13.27). By the hypothesis $d \geq \delta n^{1 / \beta}$, we have

$$
k \simeq\left(\frac{d^{\beta}}{n}\right)^{1 /(\beta-1)} .
$$

Condition (13.20) follows from the hypothesis $n \geq \varepsilon^{-1} d$, provided $\varepsilon$ is small enough. From (13.28), (13.21), and (13.25), we obtain

$$
m \simeq\left(\frac{n}{d}\right)^{\beta /(\beta-1)} \quad \text { and } \quad r \simeq\left(\frac{n}{d}\right)^{1 /(\beta-1)} .
$$

Hence, by (13.26) and $m \leq n / k$,

$$
\tilde{P}_{n}(x, y) \geq c^{k} m^{-\alpha / \beta} \geq n^{-\alpha / \beta} k^{\alpha / \beta} \exp (-C k) \geq n^{-\alpha / \beta} \exp \left(-C^{\prime} k\right) .
$$

Substituting here $k$ from (13.28), we obtain (13.18).

\section{Parity matters}

Let us recall that $(L E)$ contains the estimate for $p_{n}+p_{n+1}$ rather than for $p_{n}$. In this section we discuss to what extent it is possible to estimate $p_{n}$ from below. In general, there is no lower bound for $p_{n}(x, y)$ for the parity reason. Indeed, on any bipartite graph, the length of any path from $x$ to $y$ has the same parity as $d(x, y)$. Therefore, $p_{n}(x, y)=0$ if $n \not \equiv d(x, y)(\bmod 2)$.

We immediately obtain the following result for bipartite graphs.

PROPOSITION 14.1

If $(\Gamma, \mu)$ is bipartite and satisfies $(L E)$, then

$$
p_{n}(x, y) \geq c n^{-\alpha / \beta} \exp \left(-\left(\frac{d(x, y)^{\beta}}{c n}\right)^{1 /(\beta-1)}\right)
$$


for all $x, y \in \Gamma$ and $n \geq 1$ such that

$$
n \geq d(x, y) \quad \text { and } \quad n \equiv d(x, y) \quad(\bmod 2) .
$$

\section{Proof}

Indeed, assuming (14.2), $n+1$ and $d(x, y)$ have different parities whence $p_{n+1}(x, y)=0$, and (14.1) follows from $(L E)$.

If there is enough "mixing of parity" in the graph, then one does get the lower bound regardless of the parity of $n$ and $d(x, y)$.

\section{PROPOSITION 14.2}

Assume that graph $(\Gamma, \mu)$ satisfies $\left(p_{0}\right),(L E)$, and the following "mixing” condition: there is an odd positive integer $n_{0}$ such that

$$
\inf _{x \in \Gamma} P_{n_{0}}(x, x)>0 .
$$

Then lower bound (14.1) holds for all $n>n_{0}$ and $x, y \in \Gamma$, provided $n \geq d(x, y)$.

For example, if $n_{0}=1$, then hypothesis (14.3) means that each point $x \in \Gamma$ has a loop edge $\overline{x x}$. If $n_{0}=3$ and there are no loops, then (14.3) means that, for each point $x \in \Gamma$, there is an edge triangle $\overline{x y}, \overline{y z}, \overline{z x}$. This property holds, in particular, for the graphical Sierpiński gasket (see Figure 1).

\section{Proof}

By (9.2) we obtain, for any positive integer $m$,

$$
p_{2 m}(x, x) \geq \frac{1}{V(x, m+1)}\left(\sum_{z \in B(x, m+1)} p_{m}(x, z) \mu(z)\right)^{2}=\frac{1}{V(x, m+1)} .
$$

Condition $\left(p_{0}\right)$ and Proposition 3.1 imply $V(x, m+1) \leq C^{m+1} \mu(x)$, whence

$$
P_{2 m}(x, x)=p_{2 m}(x, x) \mu(x) \geq C^{-m-1} .
$$

Since we use this lower estimate only for the bounded range of $m \leq m_{0}$, we can rewrite it as

$$
P_{2 m}(x, x) \geq c,
$$

where $c=c\left(m_{0}\right)>0$.

Assuming $n>n_{0}$, we have, by semigroup property (5.15),

$$
p_{n}(x, y)=\sum_{z \in \Gamma} p_{n-n_{0}}(x, z) P_{n_{0}}(z, y) \geq p_{n-n_{0}}(x, y) P_{n_{0}}(y, y)
$$




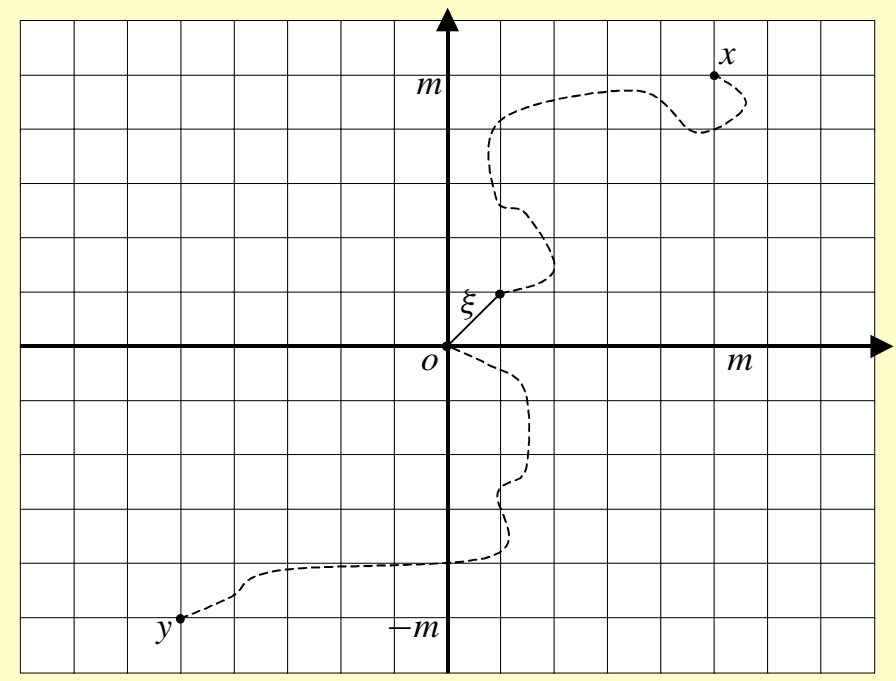

Figure 10. Every path of odd length from $x$ to $y$ goes through $o$ and $\xi$

and, in the same way,

$$
p_{n}(x, y) \geq p_{n-n_{0}+1} P_{n_{0}-1}(y, y) .
$$

By hypothesis (14.3), we can estimate $P_{n_{0}}(y, y)$ from below by a positive constant. Also, $P_{n_{0}-1}(y, y)$ is bounded below by a constant, as in (14.4). Hence, adding up (14.5) and (14.6), we obtain

$$
p_{n}(x, y) \geq c\left(p_{n-n_{0}}(x, y)+p_{n-n_{0}+1}(x, y)\right) .
$$

The right-hand side of (14.7) can be estimated from below by $(L E)$, whence (14.1) follows.

Finally, let us show an example that explains why in general one cannot replace $p_{n}+$ $p_{n+1}$ in $(L E)$ by $p_{n}$, even assuming the parity condition $n \equiv d(x, y)(\bmod 2)$.

\section{Example 14.1}

Let $(\Gamma, \mu)$ be $\mathbb{Z}^{D}$ with the standard weight $\mu_{x y}=1$ for $x \sim y$, and let $D>4$. We modify $\Gamma$ by adding one more edge $\xi$ of weight 1 , which connects the origin $o=(0,0, \ldots, 0)$ to the point $(1,1,0,0, \ldots, 0)$, and we denote the new graph by $\left(\Gamma^{\prime}, \mu^{\prime}\right)$.

Clearly, the volume growth and the Green kernel on $\left(\Gamma^{\prime}, \mu^{\prime}\right)$ are of the same order 
as on $(\Gamma, \mu)$; that is,

$$
V(x, r) \simeq r^{D} \quad \text { and } \quad g(x, y) \simeq d(x, y)^{2-D} .
$$

Hence, for both graphs one has, by Theorem 2.1,

$$
p_{n}(x, y) \leq C n^{-D / 2} \exp \left(-\frac{d^{2}(x, y)}{C n}\right)
$$

and a similar lower bound $(L E)$ for $p_{n}+p_{n+1}$. Since $\mathbb{Z}^{D}$ is bipartite, we have for $(\Gamma, \mu)$, by Proposition 14.1,

$p_{n}(x, y) \geq c n^{-D / 2} \exp \left(-\frac{d^{2}(x, y)}{c n}\right) \quad$ if $n \geq d(x, y)$ and $n \equiv d(x, y) \quad(\bmod 2)$.

Let us show that $\left(\Gamma^{\prime}, \mu^{\prime}\right)$ does not satisfy (14.9). Fix some (large) odd integer $m$, and consider points $x=(m, m, 0,0, \ldots, 0)$ and $y=-x$ (see Figure 10).

The distance $d(x, y)$ on $\Gamma$ is equal to $4 m$, whereas the distance $d^{\prime}(x, y)$ on $\Gamma^{\prime}$ is $4 m-1$, due to the shortcut $\xi$. Denote $n=m^{2}$. Then $n \equiv d^{\prime}(x, y)(\bmod 2)$ and $n>d^{\prime}(x, y)$. Let us estimate from above $p_{n}(x, y)$ on $\left(\Gamma^{\prime}, \mu^{\prime}\right)$ and show that it does not satisfy lower bound (14.9). Since $n$ is odd and all odd paths from $x$ to $y$ have to go through the edge $\xi$, the strong Markov property yields

$$
p_{n}(x, y)=\sum_{k=0}^{n} \mathbb{P}_{x}(\tau=k) p_{n-k}(o, y),
$$

where $\tau$ is the first time the random walk hits the point $o$. If $n-k<m$, then $p_{n-k}(o, y)=0$. If $n-k \geq m$, then we estimate $p_{n-k}(o, y)$ by (14.8) as follows:

$$
p_{n-k}(o, y) \leq \frac{C}{(n-k)^{D / 2}} \leq \frac{C}{m^{D / 2}} .
$$

Therefore, (14.10) implies

$$
p_{n}(x, y) \leq C m^{-D / 2} \mathbb{P}_{x}\{\tau<\infty\} .
$$

The $\mathbb{P}_{x}$-probability to hit $o$ is of the order $g(x, o) \simeq m^{2-D}$. Hence, we obtain

$$
p_{n}(x, y) \leq C m^{-(3 D / 2-2)}=C n^{-(3 D / 4-1)}=o\left(n^{-D / 2}\right)
$$

so that lower bound (14.9) cannot hold.

A more careful argument shows that, in fact, $p_{n}(x, y) \simeq n^{-(D-1)}$. 


\section{Consequences of the heat kernel estimates}

Here we prove the remaining part of Theorem 2.1, as stated in the next proposition.

PROPOSITION 15.1

Assuming $\left(p_{0}\right)$, we have

$$
(L E)+(U E) \Longrightarrow(V)+(G) .
$$

\section{Proof}

The Green kernel is related to the heat kernel by

$$
g(x, y)=\sum_{n=0}^{\infty} p_{n}(x, y) .
$$

Let $x \neq y$. Then $p_{0}(x, y)=0$, and the upper bound $(U E)$ for $p_{n}$ implies the upper bound for $g$ as follows:

$$
g(x, y) \leq C \sum_{n=1}^{\infty} n^{-\alpha / \beta} \exp \left(-c\left(\frac{d^{\beta}}{n}\right)^{1 /(\beta-1)}\right),
$$

where $d=d(x, y)$. By estimating the sum via an integral, we obtain $g(x, y) \leq C d^{-\gamma}$ with $\gamma=\alpha-\beta$. Similarly, one proves $g(x, y) \leq C d^{-\gamma}$ using $(L E)$ and the obvious consequence of (15.1):

$$
g(x, y) \geq \frac{1}{2} \sum_{n=1}^{\infty}\left(p_{n}(x, y)+p_{n+1}(x, y)\right) .
$$

Let us prove the upper bound for the volume

$$
V(x, R) \leq C R^{\alpha}
$$

for any $x \in \Gamma$ and $R \geq 1$. Indeed, for any $n \in \mathbb{N}$, we have

$$
\sum_{y \in \Gamma} p_{n}(x, y) \mu(y) \equiv 1,
$$

whence

$$
\sum_{y e B(x, R)}\left(p_{n}(x, y)+p_{n+1}(x, y)\right) \mu(y) \leq 2
$$

and

$$
V(x, R) \leq 2\left(\inf _{y \in B(x, R)}\left(p_{n}(x, y)+p_{n+1}(x, y)\right)\right)^{-1} .
$$


Taking $n \simeq R^{\beta}$ and applying ( $\left.L E\right)$, we see that the inf is bounded below by $c n^{-\alpha / \beta} \simeq$ $R^{-\alpha}$ whence $(V \leq)$ follows.

Let us prove the lower bound for the volume

$$
V(x, R) \geq c R^{\alpha} .
$$

We first show that $(U E)$ and $(V \leq)$ imply the following inequality:

$$
\sum_{y \notin B(x, R)} p_{n}(x, y) \mu(y) \leq \frac{1}{2}, \quad \forall n \leq \varepsilon R^{\beta},
$$

provided $\varepsilon>0$ is sufficiently small. Denoting $R_{k}=2^{k} R$, we have

$$
\begin{aligned}
\sum_{y \notin B(x, R)} p_{n}(x, y) \mu(y) & \leq C \sum_{y \notin B(x, R)} n^{-\alpha / \beta} \exp \left[-c\left(\frac{d(x, y)^{\beta}}{n}\right)^{1 /(\beta-1)}\right] \\
& \leq C \sum_{k=0}^{\infty} \sum_{y \in B\left(x, R_{k+1}\right) \backslash B\left(x, R_{k}\right)} n^{-\alpha / \beta} \exp \left[-c\left(\frac{R_{k}^{\beta}}{n}\right)^{1 /(\beta-1)}\right] \\
& \leq C \sum_{k=0}^{\infty} R_{k}^{\alpha} n^{-\alpha / \beta} \exp \left[-c\left(\frac{R_{k}^{\beta}}{n}\right)^{1 /(\beta-1)}\right] \\
& =C \sum_{k=0}^{\infty}\left(\frac{2^{k} R}{n^{1 / \beta}}\right)^{\alpha} \exp \left[-c\left(\frac{2^{k} R}{n^{1 / \beta}}\right)^{\beta /(\beta-1)}\right]
\end{aligned}
$$

If $R / n^{1 / \beta}$ is large enough, then the right-hand side of (15.4) is majorized by a geometric series, and the sum can be made arbitrarily small, in particular, smaller than $1 / 2$.

From (15.2) and (15.3), we conclude that

$$
\sum_{y \in B(x, R)} p_{n}(x, y) \mu(y) \geq \frac{1}{2},
$$

whence

$$
V(x, R) \geq \frac{1}{2}\left(\sup _{y \in B(x, R)} p_{n}(x, y)\right)^{-1} .
$$

Finally, choosing $n=\left[\varepsilon R^{\beta}\right]$ and using the upper bound $p_{n}(x, y) \leq C n^{-\alpha / \beta}$, we obtain $(V \geq)$.

This argument works only if $\varepsilon R^{\beta} \geq 1$. Let us now prove ( $V \geq$ ) for the opposite case when $\varepsilon R^{\beta}<1$. To that end, define $R_{0}$ by $\varepsilon R_{0}^{\beta}=1$. Then we have $R<R_{o}$. By hypothesis $\left(p_{0}\right)$ and Proposition 3.1, we have $V\left(x, R_{0}\right) \leq C \mu(x)$. Combining with the lower bound $(V \geq)$ for $V\left(x, R_{0}\right)$, we obtain $\mu(x) \geq c>0$. In particular, for any $R>0$, we have $V(x, R) \geq c$, which implies $(V \geq)$ for the bounded range of $R$. 


\section{Remark 15.1}

Using a similar argument, one can also show the following implication:

$$
(V)+(U E)+(H) \Longrightarrow(L E) .
$$

Indeed, as we have seen in the proof of Proposition 15.1, $(U E)$ implies $(G \leq)$, which, together with $(V)$, is enough to obtain $(E \leq)$ (see Proposition 6.3). From $(U E)$ and $(V)$, one obtains the diagonal lower bound $p_{2 n}(x, x) \geq c n^{-\alpha / \beta}$. Indeed, from (9.2) and (15.5) with $R=C n^{1 / \beta}$, we deduce

$$
p_{2 n}(x, x) \geq \frac{1}{V(x, R)}\left(\sum_{y \in B(x, R)} p_{n}(x, y) d \mu(y)\right)^{2} \geq \frac{1}{4 V(x, R)} \simeq n^{-\alpha / \beta} .
$$

From this estimate, one gets $(D L E)$ (see [56]; the argument is similar to the proof of (6.6)). Also, $(D U E)$ follows trivially from $(U E)$. Hence, having ( $D U E),(D L E)$, $(E \leq)$, and $(H)$, we obtain $(N L E)$ by Proposition 13.1, and then we deduce $(L E)$ from $(N L E)+(V)$ by Proposition 13.2.

Implication (15.6) yields that $(V)+(U E)+(H)$ is equivalent to either of our main conditions $(V)+(G)$ and $(U E)+(L E)$. Indeed, we have

$$
(V)+(G) \Longrightarrow(V)+(U E)+(H) \Longrightarrow(U E)+(L E),
$$

where the first implication follows by Theorem 2.1 and Proposition 10.1, and the second is the same as (15.6). We are left to close the circle by Theorem 2.1 or Proposition 15.1 .

\section{Appendix. The list of the lettered conditions}

Here we provide a list of the lettered conditions frequently used in the paper. The relations among the exponents $\alpha, \beta, \gamma, v$ are as follows:

$$
\alpha>\beta \geq 2, \quad \gamma=\alpha-\beta, \quad \text { and } \quad v=\alpha / \beta .
$$

In all conditions, $n$ is an arbitrary positive integer, $R$ is an arbitrary positive real number, and $x, y$ are arbitrary points on $\Gamma$, subject to additional restrictions if any. The constants $C, c, \delta, \varepsilon, p_{0}$ are positive. We have the following list:

$$
\begin{gathered}
V(x, R) \simeq R^{\alpha}, \quad \forall R \geq 1, \\
E(x, R) \simeq R^{\beta}, \quad \forall R \geq 1, \\
g(x, y) \simeq d(x, y)^{-\gamma}, \quad x \neq y,
\end{gathered}
$$




$$
\begin{aligned}
& V(x, 2 R) \leq C V(x, R), \\
& \bar{E}(x, R) \leq C E(x, R),
\end{aligned}
$$

$\lambda_{1}(A) \geq c \mu(A)^{-1 / v} \quad$ for all nonempty finite sets $A \subset \Gamma$,

$$
p_{n}(x, x) \leq C n^{-1 / v},
$$

$$
p_{n}(x, y) \leq C n^{-\alpha / \beta} \exp \left[-\left(\frac{d(x, y)^{\beta}}{C n}\right)^{1 /(\beta-1)}\right]
$$

$\left(p_{n}+p_{n+1}\right)(x, y) \geq c n^{-\alpha / \beta} \exp \left[-\left(\frac{d(x, y)^{\beta}}{c n}\right)^{1 /(\beta-1)}\right] \quad$ if $n \geq d(x, y)$,

$$
p_{2 n}^{B(x, R)}(x, x) \geq c n^{-\alpha / \beta} \quad \text { if } n \leq \varepsilon R^{\beta},
$$

$$
p_{n}(x, y)+p_{n+1}(x, y) \geq c n^{-\alpha / \beta} \quad \text { if } d(x, y) \leq \delta n^{1 / \beta},
$$

$$
\begin{gathered}
\Psi_{n}(x, R):=\mathbb{P}_{x}\left(T_{B(x, R)} \leq n\right) \leq C \exp \left[-\left(\frac{R^{\beta}}{C n}\right)^{1 /(\beta-1)}\right], \\
P(x, y) \geq p_{0} \quad \text { if } x \sim y,
\end{gathered}
$$

$$
\max _{B(x, R)} u \leq H \min _{B(x, R)} u
$$

for any function $u$ nonnegative in $\bar{B}(x, 2 R)$ and harmonic in $B(x, 2 R)$.

\section{References}

D. G. ARONSON, Non-negative solutions of linear parabolic equations, Ann. Scuola Norm. Sup. Pisa (3) 22 (1968), 607-694, MR 55:8553; Addendum, Ann. Scuola Norm. Sup. Pisa (3) 25 (1971), 221-228. MR 55:8554 467 P. AUSCHER, Regularity theorems and heat kernel for elliptic operators, J. London Math. Soc. (2) 54 (1996), 284-296. MR 97f:35034 467 regularity, Ann. Inst. H. Poincaré Probab. Statist. 35 (1999), 605-630. 
[4] M. T. BARLOW, "Diffusions on fractals" in Lectures on Probability Theory and Statistics (Saint-Flour, France, 1995), Lecture Notes in Math. 1690, Springer, Berlin, 1998, 1-121. MR 2000a:60148 452, 458, 459

M. T. BARLOW and R. F. BASS, The construction of Brownian motion on the Sierpinski carpet, Ann. Inst. H. Poincaré Probab. Statist. 25 (1989), 225-257. MR 91d:60183 465

\section{- Transition densities for Brownian motion on the Sierpiński carpet, Probab.} Theory Related Fields 91 (1992), 307-330. MR 93k:60203 465, 467
_ Brownian motion and harmonic analysis on Sierpinski carpets, Canad. J. Math. 51 (1999), 673-744. MR 2000i:60083 453, 459, 465, 467

_ , "Random walks on graphical Sierpinski carpets" in Random Walks and Discrete Potential Theory (Cortona, Italy, 1997), Sympos. Math. 39, Cambridge Univ. Press, Cambridge, 1999, 26-55. MR CMP 1802425 453, 458, 459, 467 M. T. BARLOW, T. COULHON, and A. GRIGOR'YAN, Manifolds and graphs with slow

heat kernel decay, to appear in Invent. Math. 458, 464
M. T. BARLOW and E. A. PERKINS, Brownian motion on the Sierpinski gasket, Probab. Theory Related Fields 79 (1988), 543-623. MR 89g:60241 453

I. BENJAMINI, I. CHAVEL, and E. A. FELDMAN, Heat kernel lower bounds on Riemannian manifolds using the old ideas of Nash, Proc. London Math. Soc. (3) 72 (1996), 215-240. MR 97c:58150 467
A. BOUKRICHA, Das Picard-Prinzip und verwandte Fragen bei Störung von harmonischen Räumen, Math. Ann. 239 (1979), 247-270. MR 81h:31018 467, 486

E. A. CARLEN, S. KUSUOKA, and D. W. STROOCK, Upper bounds for symmetric Markov transition functions, Ann. Inst. H. Poincaré Probab. Statist. 23 (1987), 245-287. MR 88i:35066 467, 470

G. CARRON, "Inégalités isopérimétriques de Faber-Krahn et conséquences" in Actes de la table ronde de géométrie différentielle (Luminy, France, 1992), Semin. Congr. 1, Soc. Math. France, Montrouge, 1996, 205-232. MR 97m:58198 465, 469
I. CHAVEL, Eigenvalues in Riemannian Geometry, Pure Appl. Math. 115, Academic Press, Orlando, 1984. MR 86g:58140 452
- Isoperimetric inequalities and heat diffusion on Riemannian manifolds, lecture notes, 1999. 452
S. Y. CHENG, P. LI, and S.-T. YAU, On the upper estimate of the heat kernel of a complete Riemannian manifold, Amer. J. Math. 103 (1981), 1021-1063. MR 83c:58083 467, 491

S. Y. CHENG and S.-T. YAU, Differential equations on Riemannian manifolds and their geometric applications, Comm. Pure Appl. Math. 28 (1975), 333-354. MR 52:6608 459

F. R. K. CHUNG, Spectral Graph Theory, CBMS Regional Conf. Ser. in Math. 92, Amer. Math. Soc., Providence, 1997. MR 97k:58183 460

T. COULHON, Ultracontractivity and Nash type inequalities, J. Funct. Anal. 141 (1996), 510-539. MR 97j:47055 469

T. COULHON and A. GRIGOR'YAN, On-diagonal lower bounds for heat kernels and 
Markov chains, Duke Math. J. 89 (1997), 133-199. MR 98e:58159 467

[22] - Random walks on graphs with regular volume growth, Geom. Funct. Anal. 8 (1998), 656-701. MR 99e:60153 458, 460, 471

[23] T. COULHON and L. SALOFF-COSTE, Puissances d'un opérateur régularisant, Ann. Inst. H. Poincaré Probab. Statist. 26 (1990), 419-436. MR 91j:43002 467

Related Fields 97 (1993), 423-431. MR 95b:60085 467

\section{E. B. DAVIES, Heat Kernels and Spectral Theory, Cambridge Tracts in Math. 92,} Cambridge Univ. Press, Cambridge, 1989. MR 90e:35123 452
- Pointwise bounds on the space and time derivatives of heat kernels, J. Operator Theory 21 (1989), 367-378. MR 90k:58214 467
—, Non-Gaussian aspects of heat kernel behaviour, J. London Math. Soc. (2) 55 (1997), 105-125. MR 97i:58169 467

T. DELMOTTE, Parabolic Harnack inequality and estimates of Markov chains on graphs, Rev. Mat. Iberoamericana 15 (1999), 181-232. MR 2000b:35103 453, 458

E. B. FABES and D. W. STROOCK, A new proof of Moser's parabolic Harnack inequality using the old ideas of Nash, Arch. Rational Mech. Anal. 96 (1986), 327-338. MR 88b:35037 467

S. GOLDSTEIN, "Random walks and diffusion on fractals" in Percolation Theory and Ergodic Theory of Infinite Particle Systems (Minneapolis, 1984/85), ed. H. Kesten, IMA Vol. Math. Appl. 8, Springer, New York, 1987, 121-129. MR 88g:60245 453

A. A. GRIGOR'YAN, The heat equation on non-compact Riemannian manifolds (in Russian), Mat. Sb. 182, no. 1 (1991), 55-87; English translation in Math. USSR-Sb. 72, no. 1 (1992), 47-77. MR 92h:58189 453 - Heat kernel upper bounds on a complete non-compact manifold, Rev. Mat. Iberoamericana 10 (1994), 395-452. MR 96b:58107 465, 469

_ Integral maximum principle and its applications, Proc. Roy. Soc. Edinburgh Sect. A 124 (1994), 353-362. MR 95c:35045 465

- Upper bounds of derivatives of the heat kernel on an arbitrary complete manifold, J. Funct. Anal. 127 (1995), 363-389. MR 96a:58183 467

_ , "Estimates of heat kernels on Riemannian manifolds" in Spectral Theory and Geometry (Edinburgh, 1998), ed. B. Davies and Yu. Safarov, London Math. Soc. Lecture Note Ser. 273, Cambridge Univ. Press, Cambridge, 1999, 140-225. MR 2001b:58040 452

B. M. HAMBLY and T. KUMAGAI, Transition density estimates for diffusion processes on post critically finite self-similar fractals, Proc. London Math. Soc. (3) 78 (1999), 431-458. MR 99m:60118 453

W. HEBISCH and L. SALOFF-COSTE, Gaussian estimates for Markov chains and random walks on groups, Ann. Probab. 21 (1993), 673-709. MR 94m:60144 452, 458

- On the relation between elliptic and parabolic Harnack inequalities, preprint, 2000. 467, 468 
[39] O. D. JONES, Transition probabilities for the simple random walk on the Sierpinski graph, Stochastic Process. Appl. 61 (1996), 45-69. MR 97b:60115 453, 458

[40] J. KIGAMI, Harmonic calculus on p.c.f. self-similar sets, Trans. Amer. Math. Soc. 335 (1993), 721-755. MR 93d:39008 453

[41] - Harmonic calculus on limits of networks and its application to dendrites, $\mathrm{J}$. Funct. Anal. 128 (1995), 48-86. MR 96e:60130 453

[42] J. KIGAMI and M. L. LAPIDUS, Weyl's problem for the spectral distribution of Laplacians on p.c.f. self-similar fractals, Comm. Math. Phys. 158 (1993), 93-125. MR 94m:58225 453

[43] N. V. KRYLOV and M. V. SAFONOV, A certain property of solutions of parabolic equations with measurable coefficients (in Russian), Izv. Akad. Nauk SSSR Ser. Mat. 44, no. 1 (1980), 161-175, 239; English translation in Math. USSR-Izv. 16 (1981), 151-164. MR 83c:35059 467

[44] T. KUMAGAI, Estimates of transition densities for Brownian motion on nested fractals, Probab. Theory Related Fields 96 (1993), 205-224. MR 94e:60068 453

S. KUSUOKA and Z. X. YIN [X. Y. ZHOU], Dirichlet forms on fractals: Poincaré constant and resistance, Probab. Theory Related Fields 93 (1992), 169-196. MR 94e:60069 453

E. M. LANDIS, The Second Order Equations of Elliptic and Parabolic Type (in Russian), Izdat. "Nauka," Moscow, 1971, MR 47:9044; English translation in Transl. Math. Monogr. 171, Amer. Math. Soc., Providence, 1998. MR 98k:35034 467

P. LI and S.-T. YAU, On the parabolic kernel of the Schrödinger operator, Acta Math. 156 (1986), 153-201. MR 87f:58156 452

F. LUST-PIQUARD, Lower bounds on $\left\|K^{n}\right\|_{1 \rightarrow \infty}$ for some contractions $K$ of $L^{2}(\mu)$, with applications to Markov operators, Math. Ann. 303 (1995), 699-712. MR 96m:47055 458, 467

J. MOSER, On Harnack's theorem for elliptic differential equations, Comm. Pure Appl. Math. 14 (1961), 577-591. MR 28:2356 467

— A Harnack inequality for parabolic differential equations, Comm. Pure Appl. Math. 17 (1964), 101-134, MR 28:2357; Correction, Comm. Pure Appl. Math. 20 (1967), 231-236. MR 34:3121 467

J. NASH, Continuity of solutions of parabolic and elliptic equations, Amer. J. Math. 80 (1958), 931-954. MR 20:6592 470

F. O. PORPER and S. D. ĖIIDEL'MAN, Two-side estimates of fundamental solutions of second-order parabolic equations and some applications (in Russian), Uspekhi Mat. Nauk 39, no. 3 (1984), 107-156; English translation in Russian Math. Surveys 39, no. 3 (1984), 119-179. MR 86b:35078 452

L. SALOFF-COSTE, A note on Poincaré, Sobolev, and Harnack inequalities, Internat. Math. Res. Notices 1992, 27-38. MR 93d:58158 453

_ Isoperimetric inequalities and decay of iterated kernels for almost-transitive Markov chains, Combin. Probab. Comput. 4 (1995), 419-442. MR 97c:60171 458 
Notes Geom. Topology 1, International Press, Cambridge, Mass., 1994.

MR 97d:53001 452

[56] D. W. STROOCK, "Estimates on the heat kernel for second order divergence form operators" in Probability Theory (Singapore, 1989), ed. L. H. Y. Chen, K. P. Choi, K. Hu, and J. H. Lou, de Gruyter, Berlin, 1992, 29-44. MR 93m:35092 467, 505

[57] K-T. STURM, Analysis on local Dirichlet spaces, III: The parabolic Harnack inequality, J. Math. Pures Appl. (9) 75 (1996), 273-297. MR 97k:31010 454

[58] M. TAKEDA, On a martingale method for symmetric diffusion processes and its applications, Osaka J. Math. 26 (1989), 605-623. MR 91d:60193 465

[59] A. TELCS, Random walks on graphs, electric networks and fractals, Probab. Theory Related Fields 82 (1989), 435-449. MR 90h:60065 453, 458, 465, 475, 477, 478

[60] - Spectra of graphs and fractal dimensions, I, Probab. Theory Related Fields 85 (1990), 489-497. MR 91k:60075 478

[61] - Spectra of graphs and fractal dimensions, II, J. Theoret. Probab. 8 (1995), 77-96. MR 96d:60107 478

[62] - Transition probability estimates for reversible Markov chains, Electron. Comm. Probab. 5 (2000), 29-37, http://www.math.washington.edu/ ejpecp/ MR 2001b:60088 458

[63] N. TH. VAROPOULOS, Hardy-Littlewood theory for semigroups, J. Funct. Anal. 63 (1985), 240-260. MR 87a:31011 469

[64] - Analysis on nilpotent groups, J. Funct. Anal. 66 (1986), 406-431. MR 88h:22014 452

[65] N. TH. VAROPOULOS, L. SALOFF-COSTE, and T. COULHON, Analysis and Geometry on Groups, Cambridge Tracts in Math. 100, Cambridge Univ. Press, Cambridge, 1992. MR 95f:43008 452

[66] W. WOESS, Random walks on infinite graphs and groups-a survey on selected topics, Bull. London Math. Soc. 26 (1994), 1-60. MR 94i:60081 452, 459

[67] X. Y. ZHOU, Resistance dimension, random walk dimension and fractal dimension, J. Theoret. Probab. 6 (1993), 635-652. MR 95d:60119 453

Girgor'yan

Department of Mathematics, Imperial College, 180 Queen's Gate, London SW7 2BZ, United Kingdom; a.grigoryan@ic.ac.uk

Telcs

International Management Center, Graduate School of Business, Budapest, Zrinyi u. 14, Budapest H-1051, Hungary; h197tel@ella.hu 